\title{
Proprotein convertase furin regulates osteocalcin and bone endocrine function
}

\author{
Omar AI Rifai, ${ }^{1,2}$ Jacqueline Chow, ${ }^{1}$ Julie Lacombe, ${ }^{1}$ Catherine Julien, ${ }^{1}$ Denis Faubert, ${ }^{3}$ Delia Susan-Resiga, ${ }^{4}$ Rachid Essalmani, ${ }^{4}$ \\ John W.M. Creemers, ${ }^{5}$ Nabil G. Seidah, ${ }^{4,6,7}$ and Mathieu Ferron ${ }^{1,2,6,7}$ \\ IIntegrative and Molecular Physiology Research Unit, Institut de Recherches Cliniques de Montréal (IRCM), Montréal, Québec, Canada. ${ }^{2}$ Molecular Biology Programs of the Faculty of Medicine, Université \\ de Montréal, Québec, Canada. ${ }^{3}$ Proteomics Discovery Platform and ${ }^{4}$ Biochemical Neuroendocrinology Research Unit, IRCM, Québec, Canada. ${ }^{5}$ Department of Human Genetics, KU Leuven, Leuven, Belgium. \\ ${ }^{6}$ Department of Medicine, Université de Montréal, Québec, Canada. 'Division of Experimental Medicine, McGill University, Montréal, Québec, Canada.
}

\begin{abstract}
Osteocalcin (OCN) is an osteoblast-derived hormone that increases energy expenditure, insulin sensitivity, insulin secretion, and glucose tolerance. The CDNA sequence of OCN predicts that, like many other peptide hormones, OCN is first synthesized as a prohormone (pro-OCN). The importance of pro-OCN maturation in regulating OCN and the identity of the endopeptidase responsible for pro-OCN cleavage in osteoblasts are still unknown. Here, we show that the proprotein convertase furin is responsible for pro-OCN maturation in vitro and in vivo. Using pharmacological and genetic experiments, we also determined that furin-mediated pro-OCN cleavage occurred independently of its $\gamma$-carboxylation, a posttranslational modification that is known to hamper OCN endocrine action. However, because pro-OCN is not efficiently decarboxylated and activated during bone resorption, inactivation of furin in osteoblasts in mice resulted in decreased circulating levels of undercarboxylated OCN, impaired glucose tolerance, and reduced energy expenditure. Furthermore, we show that Furin deletion in osteoblasts reduced appetite, a function not modulated by OCN, thus suggesting that osteoblasts may secrete additional hormones that regulate different aspects of energy metabolism. Accordingly, the metabolic defects of the mice lacking furin in osteoblasts became more apparent under pair-feeding conditions. These findings identify furin as an important regulator of bone endocrine function.
\end{abstract}

\section{Introduction}

It has been recently established that bone acts as an endocrine organ that secretes at least 3 hormones: fibroblast growth factor 23 (FGF23), lipocalin 2 (LCN2), and osteocalcin (OCN). Bone-forming osteoblasts are the cells that produce OCN, a hormone implicated, among other functions, in the regulation of glucose and energy metabolism (1). Accordingly, when fed a normal diet, $\mathrm{Ocn}$-deficient mice $\left(\mathrm{Ocn}^{--}\right)$exhibit reduced glucose tolerance, insulin sensitivity, and circulating insulin levels, as well as decreased energy expenditure and increased fat mass (2). Mechanistic studies conducted in cell cultures and mice showed that OCN improves glucose handling by promoting insulin secretion from $\beta$ cells $(3,4)$, by favoring glucose uptake in myofibers (5), and by increasing energy expenditure (6). Studies in mice demonstrated that OCN function in $\beta$ cells and myofibers is mediated through its binding to the $\mathrm{G}$ protein-coupled receptor family $\mathrm{C}$ group 6 member A (GPRC6A) $(3,5)$. This pathway appears to be conserved in humans, since human OCN can bind and activate human GPRC6A (7), while mutations or polymorphisms in human GPRC6A are associated with insulin resistance $(8,9)$.

OCN is a small protein (46 aa in mice and 49 aa in humans) that is uniquely produced by osteoblasts and $\gamma$-carboxylated on 3 glutamic acid residues (Glu) before it is secreted. $\gamma$-Carboxylation of OCN occurs in the ER and is mediated by $\gamma$-glutamyl carboxy-

Conflict of interest: The authors have declared that no conflict of interest exists. Submitted: February 15, 2017; Accepted: August 15, 2017. Reference information: / Clin Invest. 2017;127(11):4104-4117. https://doi.org/10.1172/JCI93437. lase (GGCX), which requires reduced vitamin $\mathrm{K}$ as an essential cofactor (10). This posttranslational modification increases the affinity of OCN for hydroxyapatite, the mineral component of bone extracellular matrix (ECM). Hence, the vast majority of OCN secreted by osteoblasts is trapped in bone ECM, in which it constitutes the most abundant noncollagenous polypeptide. Although both $\gamma$-carboxylated (Gla) and undercarboxylated (ucOCN) forms of OCN are detected in serum, most in vitro and in vivo studies concur to indicate that the endocrine function of OCN is fulfilled by ucOCN in mice and humans $(3,4,11)$. This has been recently confirmed genetically through the inactivation of $G g c x$ specifically in osteoblasts, which resulted in increased circulating levels of ucOCN and improved glucose tolerance in mice (12). Additional studies showed that osteoclasts, the bone-resorbing cells, are responsible for the partial decarboxylation and activation of OCN present in bone ECM $(13,14)$. Altogether, these observations suggested a model in which hormonally inactive $\gamma$-carboxylated OCN is produced by osteoblasts and stored in the bone ECM, before being activated and released as ucOCN by osteoclasts during the process of bone resorption.

As is the case for many other peptide hormones, the sequence of Ocn cDNA predicts that it is first synthesized as a pre-prohormone composed of a signal peptide, a propeptide, and a mature hormone (Figure 1A). The propeptide of other $\gamma$-carboxylated proteins such as prothrombin and profactor IX are characterized by a recognition signal for the $\gamma$-glutamyl carboxylase (15). However, the importance of the propeptide for the $\gamma$-carboxylation and secretion of OCN has never been addressed in vivo. Moreover, it is currently unknown whether removal of the OCN propeptide depends on the $\gamma$-carbox- 
A

B

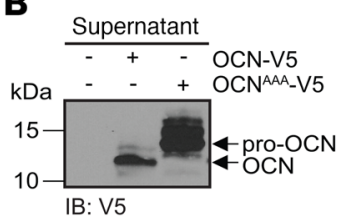

D

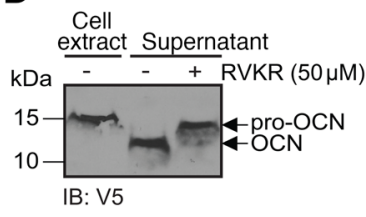

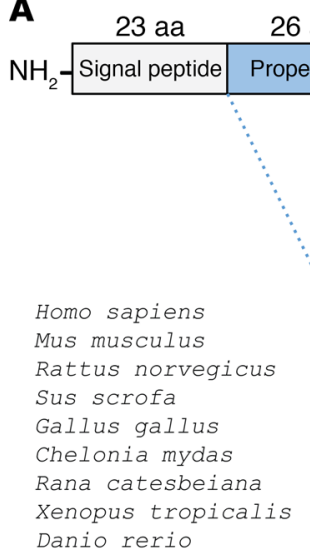

aa

$\frac{46 \text { aa }}{\ddots \ddots \text { Gature } \mathrm{OCN}}-\mathrm{COOH}$

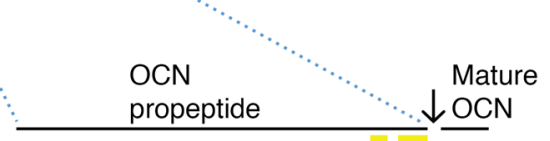

KP-SGAESK-GAAFVSKQEGSEVVKRPRR YLYQ... KP-SG--PESDKAFMSKQEGNKVVNRLRR YLGA. . . KP-SD--SESDKAFMSKQEGSKVVNRLRR YLNN . . . KP-SGADSGKGAAFVSKQEGTEVVKRLRR YLDH . . . AP-DGSDARSAKAF ISHRQRAEMVRRQKR HYAQ . . SN-SANDSPSSEAFVSKQDSAEVVRRHKR NYVY... RG-SLADSRSSEAFISRQESANFAKRHRR SNLR . . SA-SAGDSRSSEAFISRQDSANFARRLKR SYRY . . KPLSAAESPNHEGVFVKRDVAS I IMROKR AGTA... .. : : : . . * :*
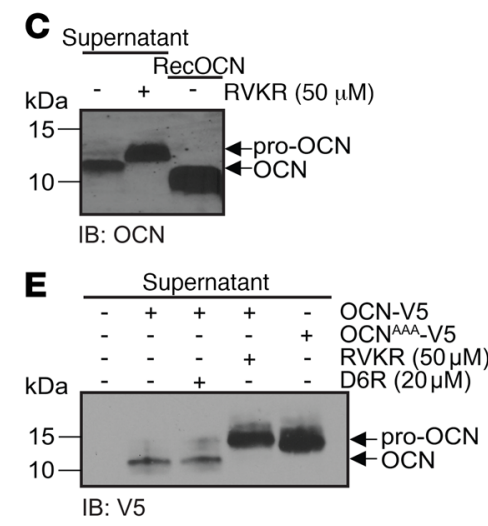

Figure 1. A PC cleaves pro-OCN at the RXRR motif in osteoblasts. (A) Schematic representation of the pre-pro-OCN protein including the approximate positions of the Gla residues, and amino acid alignment of OCN propeptide sequences from various vertebrate species: the conserved $\mathrm{RX}(\mathrm{R} / \mathrm{K}) \mathrm{R}$ motif is highlighted in yellow. Consensus symbols are included below the alignment. A single asterisk indicates a fully conserved residue; a colon indicates a strongly conserved residue; a period indicates moderate or weak conservation. (B) Western blot analysis of cell supernatant from primary osteoblasts transfected with OCN-V5 or an R46A/R48A/R49A OCN mutant (OCN $\left.{ }^{A A A}-\mathrm{V} 5\right)$, both tagged at the C-terminal with the V5 epitope. (C) Western blot analysis of endogenous OCN in the cell supernatant from differentiated mouse calvaria osteoblasts treated or not with $50 \mu \mathrm{M}$ Dec-RVKR-CMK (RVKR). (D) Western blot analysis of cell supernatant and cell extracts of CHO-IdID cells transfected with OCN-V5 and treated or not with $50 \mu \mathrm{M}$ Dec-RVKR-CMK. (E) Western blot analysis of the supernatant of primary osteoblasts transfected with $\mathrm{OCN}-\mathrm{V} 5$ or $\mathrm{OCN}{ }^{\mathrm{AAA}}-\mathrm{V} 5$ and treated or not with $50 \mu \mathrm{M}$ Dec-RVKR-CMK or $20 \mu \mathrm{M}$ D6R. IB, immunoblot.

ylation process. It should be noted that $\mathrm{OCN}$ is unique among all known $\gamma$-carboxylated proteins, as it is the only one known to be efficiently $\gamma$-carboxylated in vitro in the absence of its propeptide (16). These observations raise the question of the function of the OCN propeptide. Last, and as important, the identity of the endopeptidase responsible for processing the OCN prohormone (pro-OCN) and its importance in bone endocrine function remain unknown.

We therefore embarked on a search for the pro-OCN-converting enzyme. For that purpose, we focused on the proprotein convertases (PCs), a series of serine endoproteases that target specific motifs comprising basic residues, such as di-arginine or di-lysine, that are frequently observed in prohormone sequences (17). These enzymes function within the secretory pathway or outside the cell to cleave other proteins, either activating or inactivating them, and have been implicated in the regulation of a myriad of biological processes (18). In particular, the PCs of the subtilisin/kexin type include proprotein convertase 1 (PC1), proprotein convertase 2 (PC2), furin, proprotein convertase 4 (PC4), proprotein convertase 5A and B (PC5A and PC5B), paired basic aa-cleaving enzyme 4 (PACE4), and proprotein convertase 7 (PC7) and play critical functions in several endocrine cells by regulating the maturation and secretion of numerous peptide hormones such as insulin, glucagon, adrenocorticotropic hormone (ACTH), glucagon-like peptide 1 (GLP-1), and parathyroid hormone (PTH) $(18,19)$.

Here, we identified furin, using cell-based and genetic arguments, as the endopeptidase responsible for pro-OCN processing in osteoblasts. We also showed in cell culture and in vivo that $\gamma$-carboxylation and processing of OCN are 2 independent processes in osteoblasts. The study of mice lacking furin specifically in osteoblasts revealed that proteolysis of pro-OCN is critical for the activation of this hormone. We also found that furin may modulate energy metabolism through OCN-independent pathway(s) affecting appetite.

\section{Results}

Pro-OCN is cleaved by an intracellular PC. The putative mouse OCN propeptide exhibits a C-terminal motif consisting of 3 basic residues: Arg-Leu-Arg-Arg $\left(\mathbf{R L R R}{ }^{49}\right)$, which is highly conserved throughout bony vertebrates (Figure 1A). Because this type of basic residue-containing motif represents a consensus recognition site for PCs that is found in many secreted proteins (18), we hypothesized that pro-OCN may be cleaved by 1 or more PCs in osteoblasts. In support of this hypothesis, mutation of the RLRR sequence into ALAA resulted in a molecular shift of the OCN secreted in the culture medium of osteoblast cultures, consistent with retention of the propeptide in the mutated protein (Figure 1B). Furthermore, treatment of primary osteoblast cultures with decanoyl-RVKRchloromethyl ketone (Dec-RVKR-CMK), a cell-permeable inhibitor that blocks all 7 subtilisin/kexin-like PCs, resulted in a similar molecular shift of either endogenous OCN secreted from primary osteoblast cultures or a V5-tagged version transfected in osteoblasts or in CHO-ldlD cells (Figure 1, C-E). That Dec-RVKR-CMK treatment and the ALAA mutation induced an identical migration shift by SDS-PAGE suggests that the RLRR motif is the main, if not only, sequence present in pro-OCN that is recognized and cleaved by a PC (Figure 1E). Last, treatment of osteoblasts with hexa-Darginine (D6R), a nonpermeable inhibitor that blocks only extracellular or cell-surface PCs (20), had no impact (Figure 1E). Altogether, these results suggest that an intracellular PC in osteoblasts contributes to the conversion of pro-OCN to OCN.

Furin is the PC responsible for pro-OCN cleavage in osteoblasts. In an effort to identify the PC responsible for pro-OCN cleavage in osteoblasts, we first assessed the expression of the 8 subtilisin/ kexin-like PCs in this cell type. As shown in Figure 2A, mRNAs encoding furin (Furin) and PACE4 (Pcsk6) were expressed at high levels in this cell type, while mRNAs encoding PC7 (Pcsk7) and PC5A (Pcsk5a) were detected at lower levels. Pcsk1, Pcsk2, Pcsk5b, and Pcsk 4 mRNAs were either not detectable or expressed at very low levels in osteoblasts. Of note, the expression of both Furin and Pcsk5a was induced during osteoblast differentiation. Given these 
A

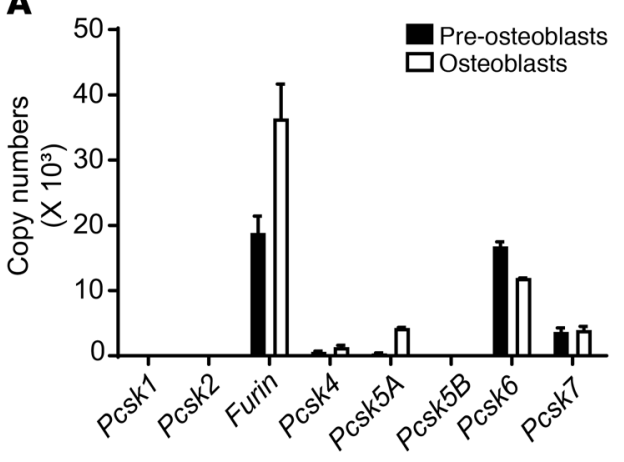

B
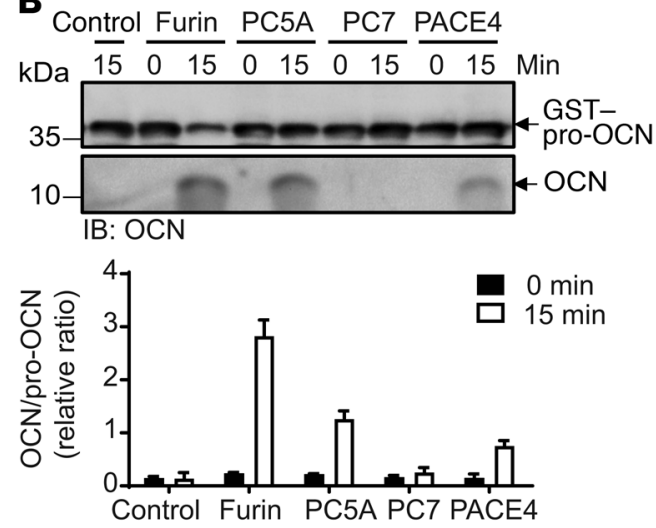

C

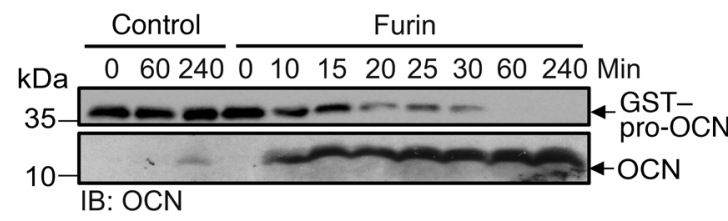

D

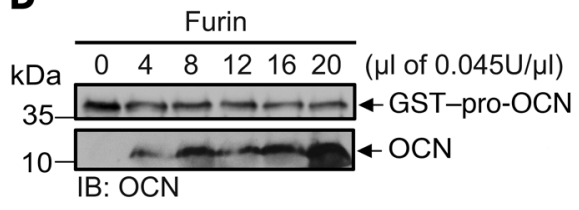

E $\frac{\text { Control Furin Control Furin }}{++++} \frac{\text { Fun }}{-- \text { GST-pro-OCN }}$ - - - + + + + pST-OCN/AA-

kDa $\begin{array}{rrrrrrrrr}0 & 30 & 0 & 30 & 0 & 30 & 0 & 30 & \text { Min }\end{array}$

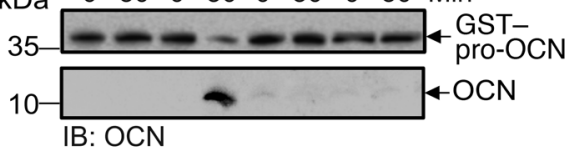

$\mathbf{F}$
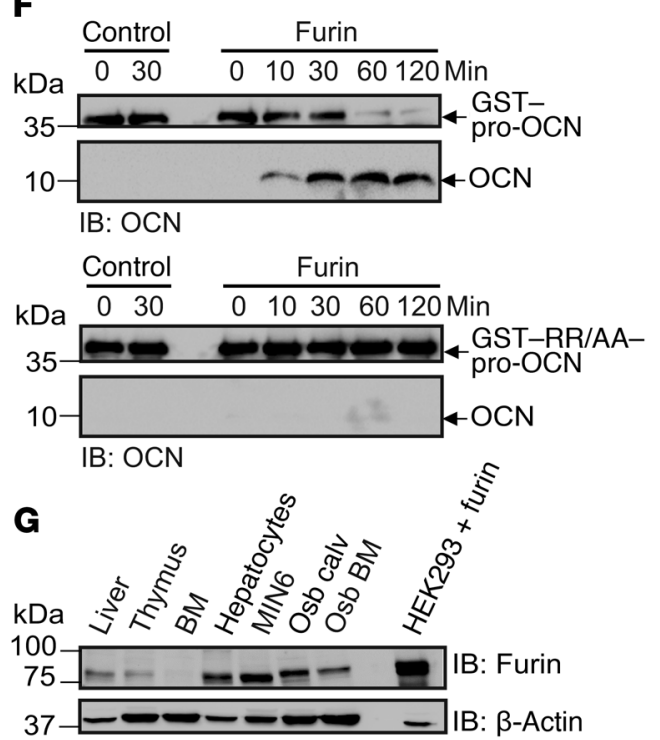

Figure 2. Furin cleaves pro-OCN in vitro. (A) Relative mRNA expression levels of kexin-like PCs in mouse nondifferentiated and differentiated osteoblasts analyzed by qPCR. Copy numbers were calculated from a standard curve prepared from mouse genomic DNA, and samples were normalized using Actb as a reference gene $(n=3)$. (B) In vitro processing assay of CST-pro-OCN incubated for 0 or 15 minutes with equal numbers of enzymatic units of furin, PC5A, PC7, and PACE4; released OCN was assessed by Western blotting, and the relative ratio of OCN/CST-pro-OCN from 3 independent experiments was quantified (graph). (C) Time course for processing of GST-pro-OCN by furin, as assessed by Western blotting. (D) In vitro processing assay of GST-pro-OCN with increasing amounts of furin for 60 minutes, as assessed by Western blotting. (E and F) In vitro processing assay of CST-pro-OCN and the R48A/R49A OCN mutant (CST-RR/AA-pro-OCN) by furin for 30 minutes (E) or for various incubation durations (F), as assessed by Western blotting. In B-F, GST-OCN and mature OCN proteins are shown as separate Western blot exposures due to the reduced transfer efficiency of mature OCN (5 kDa) compared with that of GST-pro-OCN (36 kDa). (G) Western blot analyses of furin protein expression in the indicated tissues and cell lines. Osb calv, calvaria-derived osteoblasts; Osb BM, bone marrow-derived osteoblasts; HEK293 + furin, HEK293 cells transfected with human full-length Furin cDNA. Results represent the mean \pm SEM.

results, we next tested the capacity of furin, PC5A, PC7, and PACE4 to cleave pro-OCN in vitro. Recombinant GST-pro-OCN protein produced in bacteria was incubated with the conditioned media of HEK293 cells transfected with either an empty vector or a vector expressing the soluble extracellular enzymatic domains of furin, PC5A, or PC7, or with recombinant soluble PACE4 expressed and purified from insect cells $(20,21)$. The PC activity of each conditioned media or recombinant $\mathrm{PC}$ was measured using an artificial tetrapeptide substrate prior to the assay to ensure that an equal number of enzymatic units was used (see Methods and Supplemental Figure 1A; supplemental material available online with this article; https://doi.org/10.1172/JCI93437DS1). Furin, PC5A, and PACE4, but not PC7, were able to cleave GST-pro-OCN, releasing mature OCN (Figure 2B). We noticed, however, that furin was reproducibly more efficient than were PC5A and PACE4 at cleaving pro-OCN when the incubation was performed for a short period, i.e., 15 minutes (Figure 2B). In addition, further testing revealed that furin could cleave more than $80 \%$ of pro-OCN within 30 minutes and in a dose-dependent manner (Figure 2, $\mathrm{C}$ and D). Since PC5A and PACE4 are known to act primarily on extracellular or plasma membrane-bound substrates, whereas furin acts mainly intracellularly in the secretion pathway (18), these results, combined with the observation that pro-OCN cleavage in osteoblasts was inhibited by Dec-RVKR-CMK, but not D6R (Figure 1E), suggest that pro-OCN is primarily a substrate for furin. Importantly, mutation of the RLRR motif to RLAA completely 
A

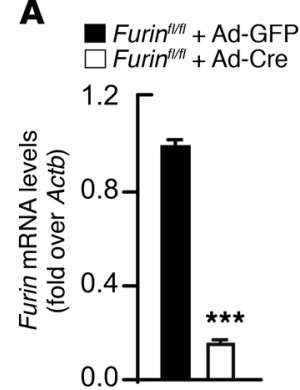

B

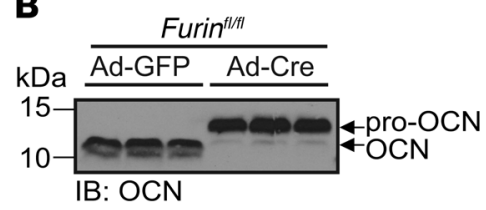

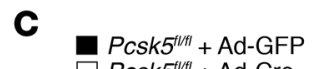

Pcsk5 $5^{t / f i}+$ Ad-Cre

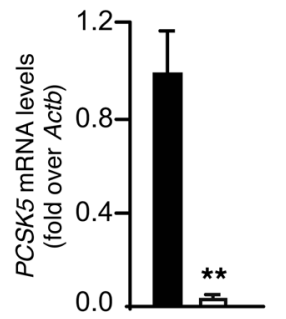

D ${ }_{15} \frac{P c s k 5^{\text {ilf }}}{\text { Ad-GFP Ad-Cre }}$
Figure 3. Furin but not PC5 is necessary for pro-OCN processing in osteoblasts. (A) Cre-mediated inactivation of Furin in Furin $^{f / f l}$ osteoblasts infected with either Ad-GFP (i.e., control osteoblasts) or Ad-Cre (i.e., Furin ${ }^{-1-}$ osteoblasts) was assessed by qPCR $(n=3)$. (B) Western blot analysis of secreted OCN from differentiated Furin fl/fl osteoblasts infected with either Ad-GFP or Ad-Cre. (C) Cre-mediated inactivation of Pcsk5 in Pcsk $5^{f / f l}$ osteoblasts infected with either Ad-GFP or Ad-Cre was assessed by qPCR $(n=3)$. (D) Western blot analysis of secreted OCN from differentiated Pcsk $5^{f l / f l}$ osteoblasts infected with either Ad-GFP or Ad-Cre. (E) Western blot analysis of secreted OCN from differentiated Furin ${ }^{f / f l}$ mouse calvaria osteoblasts infected with either Ad-GFP or Ad-Cre and treated or not with $50 \mu \mathrm{M}$ Dec-RVKR-CMK or $20 \mu \mathrm{M}$ D6R. Results represent the mean \pm SEM. ${ }^{* *} P<0.01$ and ${ }^{* *} P<0.001$, by unpaired, 2 -tailed Student's $t$ test.

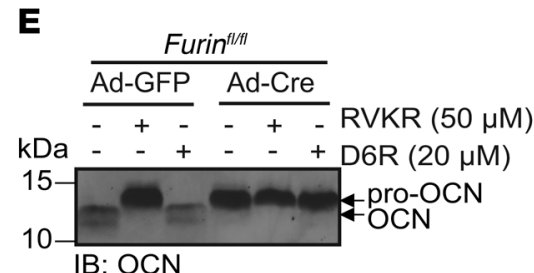

E

IB: OCN abrogated the activity of furin toward pro-OCN in vitro (Figure 2, $\mathrm{E}$ and $\mathrm{F}$ ), thus demonstrating that furin is cleaving pro-OCN specifically at this dibasic site.

Consistent with the possibility that it may play an important role in osteoblasts, furin was detected in primary osteoblasts at comparable or higher levels than those observed in hepatocytes, thymus, and MIN6 $\beta$ cells, where this enzyme is known to have nonredundant functions (Figure $2 G)(22-24)$. We therefore next assessed the requirement for furin in pro-OCN maturation in osteoblasts. To that end, Furin fl/fl $^{\prime / 2}$ primary osteoblasts were infected with adenovirus expressing either GFP (Ad-GFP) or Cre-GFP (Ad-Cre) to generate control and Furin-deficient osteoblasts (hereafter referred to as Furin ${ }^{-1}$ osteoblasts), respectively. Furin expression was significantly reduced in osteoblasts infected with Ad-Cre compared with expression levels in the control (Figure 3A). Remarkably, the inactivation of Furin in primary osteoblasts was sufficient to induce a molecular shift in the secreted OCN (Figure 3B). In contrast, the deletion of Pcsk5, the gene encoding PC5A and PC5B, in primary osteoblasts had no impact on the apparent molecular weight of OCN (Figure 3, C and D). Moreover, neither Dec-RVKR-CMK nor D6R further affected the apparent molecular weight of OCN in Furindeficient osteoblast cultures (Figure 3E), suggesting that secreted OCN retained its propeptide.

To visualize the interaction between furin and pro-OCN, mouse osteoblasts were transfected with a pro-OCN construct in which the propeptide of OCN was tagged with a $3 \mathrm{X}$ FLAG epitope at its $\mathrm{N}$-terminus, and the intracellular localization of pro-OCN and furin were assessed by immunofluorescence. As shown in Figure 4A, both the FLAG-pro-OCN and the noncleavable mutant (FLAG-pro-OCN ${ }^{\mathrm{AAA}}$ ) signals overlapped with the furin signal (middle and bottom panels), suggesting colocaliza- tion of pro-OCN with furin. However, the FLAG-pro-OCN ${ }^{\text {AAA }}$ was more abundant intracellularly than was the FLAG-pro-OCN construct (Figure 4A, middle and bottom panels). Quantification of the FLAG-pro-OCN immunofluorescence signal intensity and Western blotting confirmed an increase in the intracellular levels of pro-OCN when the furin cleavage site was mutated (Figure 4 , B and C). Consistent with the observations made using furindeficient osteoblasts (Figure 3B), FLAG-pro-OCN ${ }^{\mathrm{AAA}}$ was still secreted (Figure $4 \mathrm{C}$ ), indicating that retention of the propeptide does not prevent secretion of the protein. Altogether, these in vitro and cell biology experiments support the notion that furin is the primary, if not sole, $\mathrm{PC}$ responsible for pro-OCN processing in osteoblasts.

Pro-OCN $\gamma$-carboxylation and its processing are uncoupled in osteoblasts. In hepatocytes, the cell type in which $\gamma$-carboxylation has been the most extensively studied, this posttranslational modification of proteins occurs in the ER (10). Yet furin was shown to be active mainly in the trans-Golgi network (TGN) and beyond in several cell types $(25,26)$, thus suggesting that pro-OCN $\gamma$-carboxylation and cleavage by furin might occur sequentially in osteoblasts. To test whether these 2 processes interfere with each other, differentiated osteoblasts were treated with either warfarin, which inhibits $\gamma$-carboxylation by blocking vitamin K reduction (10), or with DecRVKR-CMK, and pro-OCN processing and $\gamma$-carboxylation were assessed. As shown in Figure 5A, warfarin efficiently blocked OCN $\gamma$-carboxylation, but did not influence its processing. Conversely, treatment with Dec-RVKR-CMK efficiently blocked pro-OCN processing, but had no significant impact on its $\gamma$-carboxylation (Figure 5B). Similarly, mutation of the 3 OCN glutamic acid (E) residues targeted by $\gamma$-carboxylation to aspartic acid (D), a mutation known to prevent this posttranslational modification in prothrombin (27), did not affect the maturation of pro-OCN to OCN 
A
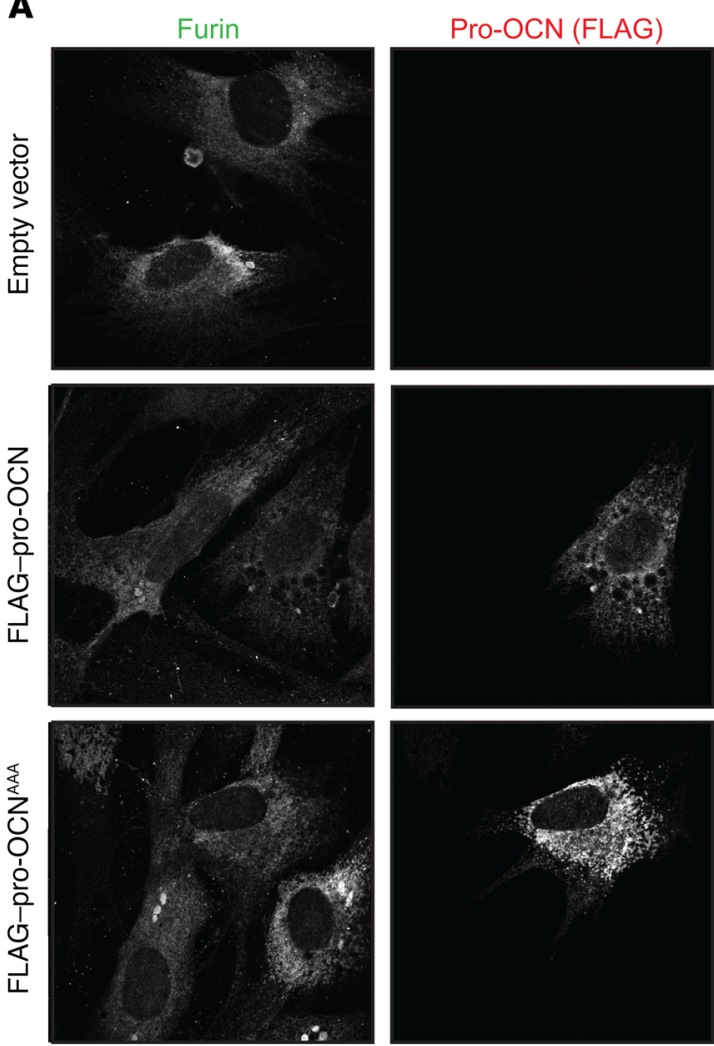

$\overline{10} \mu \mathrm{m}$
B

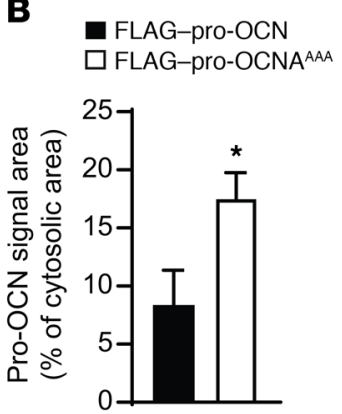

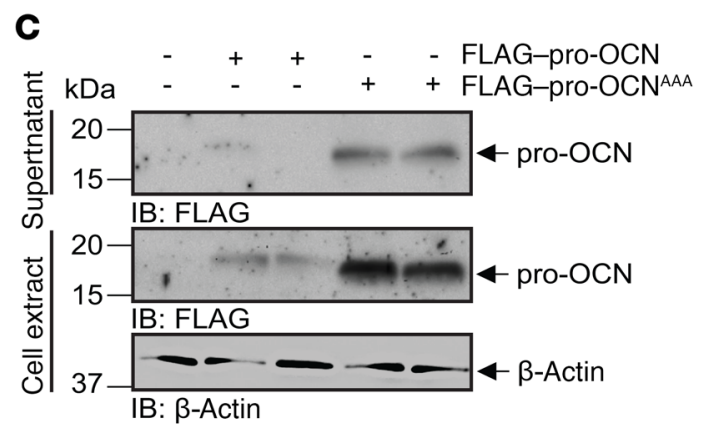

Figure 4. Furin and pro-OCN colocalize in osteoblasts. (A) Representative images of immunofluorescence analyses of primary osteoblasts transfected with FLAG-pro-OCN or the R46A/R48A/R49A FLAG-pro-OCN mutant (FLAG-pro-OCN ${ }^{\text {AAA }}$ ) expressed from the p3xFLAGMyc-CMV-23 vector. Furin is shown in green, FLAC (i.e., pro-OCN) in red, and DNA (DAPI) in blue. Scale bar: $10 \mu \mathrm{m}$. (B) Quantification of the red signal area in primary osteoblasts transfected with either FLAG-pro-OCN ( $n=9$ cells) or FLAG-pro-OCN ${ }^{\text {AAA }}(n=7$ cells). Results represent the mean \pm SEM. ${ }^{*} P<0.05$, by unpaired, 2 -tailed Student's $t$ test. (C) Western blot analysis of cell supernatant and cell extract from primary osteoblasts transfected with either FLAG-pro-OCN or FLAG-pro-OCN ${ }^{A A A}$.

in primary osteoblasts (Figure $5 \mathrm{C}$ ). We also assessed the impact of pro-OCN $\gamma$-carboxylation on its processing in vivo by studying mice lacking $G g c x$ only in osteoblasts $\left(G g c x^{f / f l}\right.$ OCN-Cre mice, hereafter referred to as $G g C x^{o s b-/-}$ mice) (12). Since the absence of $\gamma$ carboxylation prevents OCN from accumulating in the bone ECM in these mice, we evaluated OCN processing in the serum by immunoprecipitation and Western blotting. Although circulating OCN was not $\gamma$-carboxylated in $G g c x^{o s b-/-}$ mice, its migration was similar to that of OCN from control serum, indicating that OCN is processed into its mature form in vivo, even in the absence of $\gamma$-carboxylation (Figure 5D).

Finally, we sought to determine more precisely which form(s) of OCN is/are secreted by osteoblasts under normal conditions and when furin-dependent cleavage or $\gamma$-carboxylation is inhibited. For that purpose, OCN from differentiated osteoblasts was analyzed by liquid chromatography tandem mass spectrometry (LC-MS/MS) after treatment with vehicle, Dec-RVKR-CMK, or warfarin. Secreted proteins were collected, concentrated, and subjected to digestion by Arg-C, which cleaves proteins following any arginine residues. Hence, if pro-OCN was present in the medium, Arg-C would release an OCN propeptide lacking its last 3 residues, i.e., KPSGPESDKAFMSKQEGNKVVNR. Interestingly, we did not detect this peptide in the supernatant of vehicle- or warfarin-treated osteoblasts, but only in supernatant from osteoblasts treated with the PC inhibitor, together with several N-terminally truncated fragments of OCN propeptide (Table 1 and Figure $5 \mathrm{E})$. These data further indicate that pro-OCN cleavage is independent of OCN $\gamma$-carboxylation and that pro-OCN processing is fully efficient in osteoblasts, as pro-OCN is virtually absent in the medium of vehicle-treated osteoblasts. Last, since OCN propeptide is not detected in the supernatant of osteoblasts in normal conditions, this suggests that the cleaved propeptide is degraded intracellularly and is not secreted. This is consistent with an early study reporting that OCN propeptide was undetectable by radioimmunoassay in human sera or in human osteosarcoma cell supernatant (28).

Inactivation of Furin in osteoblasts results in impaired pro-OCN processing and decarboxylation in vivo. Our cell culture data indicating that furin is responsible for pro-OCN processing in osteoblasts raised the question of whether furin is also required for pro-OCN processing in vivo. To address this question, we generated mice lacking furin specifically in osteoblasts (Furin ${ }^{f / f l}$ OCN-Cre mice; hereafter referred to as Furin ${ }^{o s b-1}$ mice), since mice lacking furin in all cell types died in utero before skeletogenesis was initiated (29). For this purpose, Furin ${ }^{f l / f l}$ mice (30) were bred with $O C N$-Cre-transgenic mice, which express the Cre recombinase under the control of the human $O C N$ promoter in differentiated osteoblasts (31).

Furin $^{\text {ssb-/- }}$ mice were born at the expected Mendelian ratios and displayed no overt developmental abnormalities. We confirmed by PCR that recombination at the Furin locus occurred only in bone tissues (Figure 6A) and that the expression of furin was appreciably reduced in osteoblast cultures derived from bone marrow mesenchymal cells from Furin ${ }^{\text {osb }-/}$ mice compared with control osteoblast cultures, both at the mRNA and protein levels (Figure 6, B and C, and Supplemental Figure 1B). As 
A
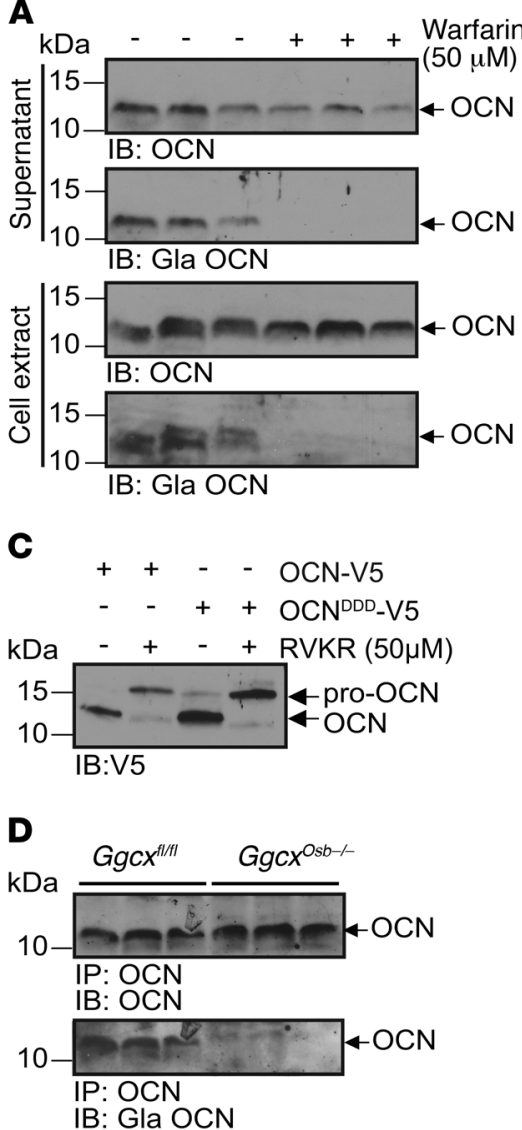

B
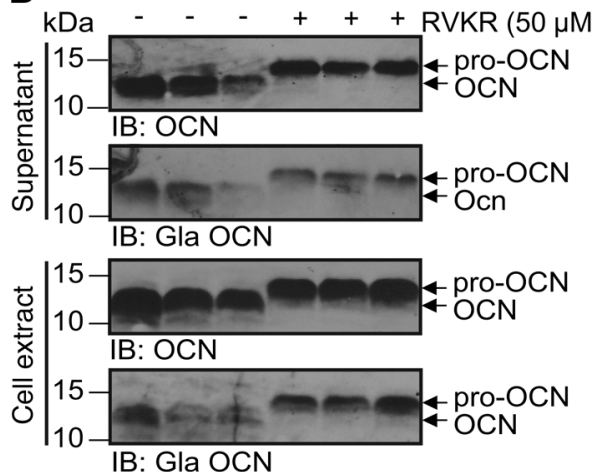

E

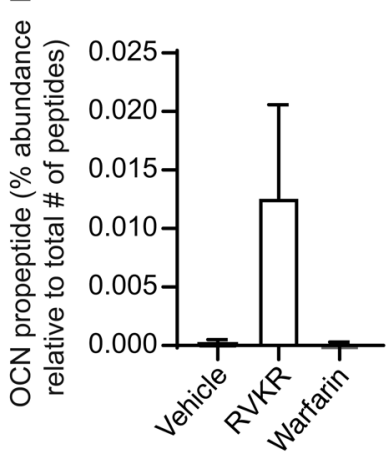

Figure 5. Pro-OCN processing and $\gamma$-carboxylation occur independently of each other in osteoblasts. (A and B) Western blot analysis of endogenous total OCN (OCN) and $\gamma$-carboxylated OCN (Gla OCN) of cell supernatant and cell extract from differentiated mouse osteoblasts treated or not with $50 \mu \mathrm{M}$ warfarin (A) or 50 $\mu M$ Dec-RVKR-CMK (RVKR) (B). (C) Western blot analysis of supernatant from osteoblasts transfected with OCN-V5 or the E13D/E17D/E20D OCN-V5 mutant (OCN $\left.{ }^{D D D}-\mathrm{V} 5\right)$ and treated or not with $50 \mu \mathrm{M}$ Dec-RVKR-CMK. (D) Western blot analysis of OCN immunoprecipitated from the serum of control mice (Ggcx fl/fi) and mice lacking $\gamma$-carboxylase in osteoblasts (Ggcx $x^{\text {osb-/- }}$ ). Total OCN and $\gamma$-carboxylated OCN were assessed by Western blotting. (E) LC-MS/MS analyses of cell supernatant of differentiated mouse osteoblasts treated or not with $50 \mu \mathrm{M}$ Dec-RVKR-CMK or $50 \mu \mathrm{M}$ warfarin. Quantification of the OCN propeptide area relative to the total peptide area in 3 independent experiments is shown (see also Table 1). Results represent the mean \pm SEM. shown in Figure 6D, the inactivation of Furin resulted in accumulation of pro-OCN and an absence of mature OCN in bones. Accordingly, OCN was only present in its proform in the serum of Furin ${ }^{o s b-/}$ mice (Figure 6E). Importantly, and confirming in vivo the observations made in osteoblast cultures, the absence of furin did not affect the level of $\gamma$-carboxylation of pro-OCN in the bone ECM, whether it was assessed by Western blotting or ELISA (Figure 6, D and F). The insulin receptor precursor (pro-INSR), another protein cleaved by furin and PACE 4 in cell culture (32) and involved in the regulation of energy metabolism by osteoblasts (33), was still processed in the bones of Furin ${ }^{o s b-1}$ mice and in Furin ${ }^{--}$osteoblasts (Supplemental Figure 1, C and D). In addition, OCN was still processed when PC5, another PC known to be expressed in osteoblasts (34) and implicated in skel-

Table 1. LC-MS/MS analyses of cell supernatant of differentiated mouse osteoblasts treated or not with $50 \mu \mathrm{M}$ Dec-RVKR-CMK (RVKR) or $50 \mu \mathrm{M}$ warfarin

\begin{tabular}{lcccc} 
Peptide sequences & \multicolumn{2}{c}{ Exclusive spectrum count } & Average Mascot \\
& Vehicle & RVKR & Warfarin & ion score \\
KPSCPESDKAFMSKQEGNKVVNR & 0 & 3 & 0 & 35.9 \\
SCPESDKAFMSKQEGNKVVNR & 0 & 7 & 0 & 41.8 \\
PESDKAFMSKQECNKVVNR & 0 & 1 & 0 & 29.2 \\
ESDKAFMSKQEGNKVVNR & 0 & 2 & 0 & 45.2
\end{tabular}

etal development (35), was inactivated in this cell type (Supplemental Figure 1E).

Interestingly, although total bone OCN content was not reduced in Furin ${ }^{\text {sb- }}$ - mice, circulating levels of total and ucOCN were decreased by almost 2-fold when assessed using ELISAs recognizing both OCN and pro-OCN (Figure 6G). These reductions were not caused by a difference in the specificity of the antibodies used in the ELISAs, since they similarly recognized pro-OCN or mature OCN under nondenaturing conditions (Supplemental Figure 1F). Because carboxylated OCN present in the bone ECM is converted to active ucOCN during the process of bone resorption (14), we hypothesized that in Furin ${ }^{\text {osb- }}$ - mice, pro-OCN was less efficiently decarboxylated and released during bone resorption compared with mature OCN. To test this possibility, RAW 264.7 preosteoclastic cells were cultured on devitalized calvaria bones isolated from con-

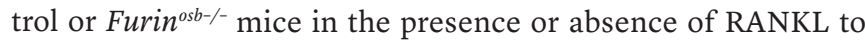
induce osteoclast differentiation. When osteoclasts were differentiated on control bones, we could detect the release of both total OCN and ucOCN, whereas when differentiated on bones isolated from Furin ${ }^{o s b-/-}$ mice, osteoclasts released less total OCN and an undetectable quantity of ucOCN (Figure 7A). Importantly, upon tartrate-resistant acid phosphatase (TRAP) staining, osteoclast differentiation did not appear to be impaired on Furin ${ }^{o s b-/}$ calvaria compared with control calvaria (Figure 7, $\mathrm{B}$ and $\mathrm{C}$ ), and the expression levels of the osteoclast-specific markers Acp5 and Clcn 7 remained unchanged in the bones of 

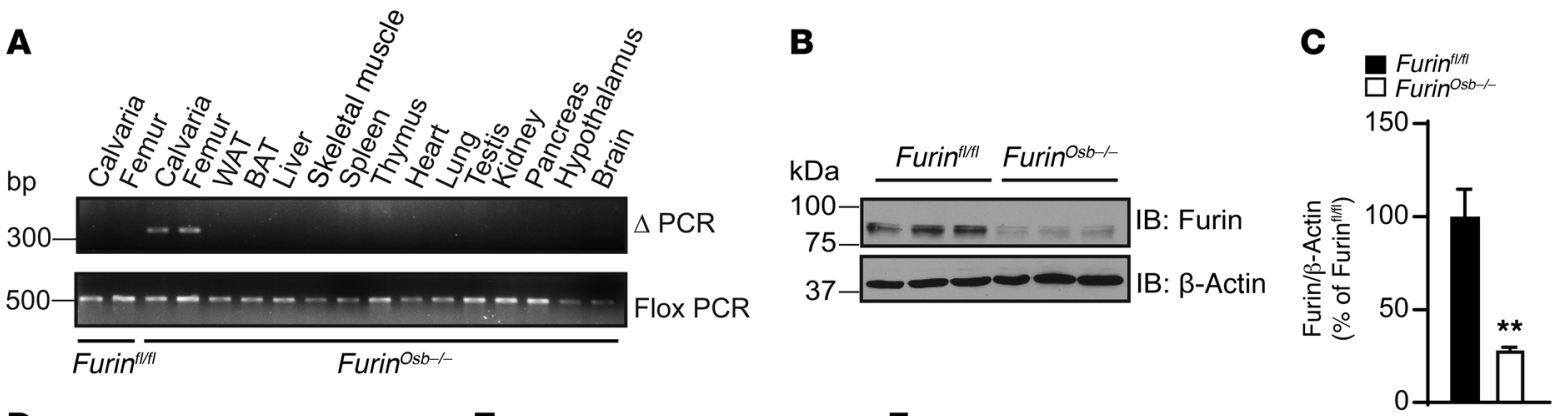

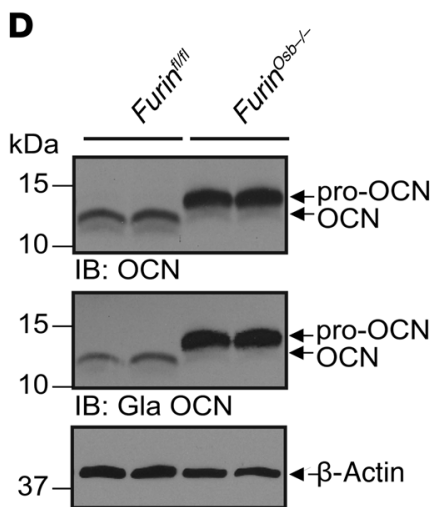

IB: $\beta$-Actin
$\mathbf{E}$

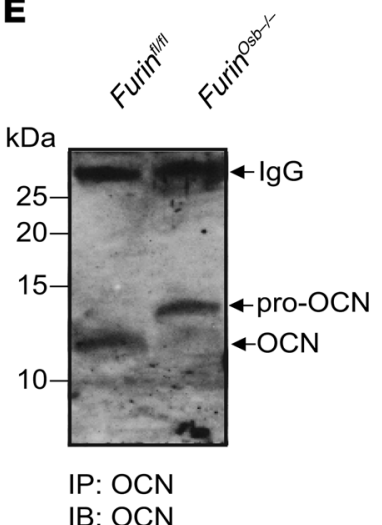

$\mathbf{F}$
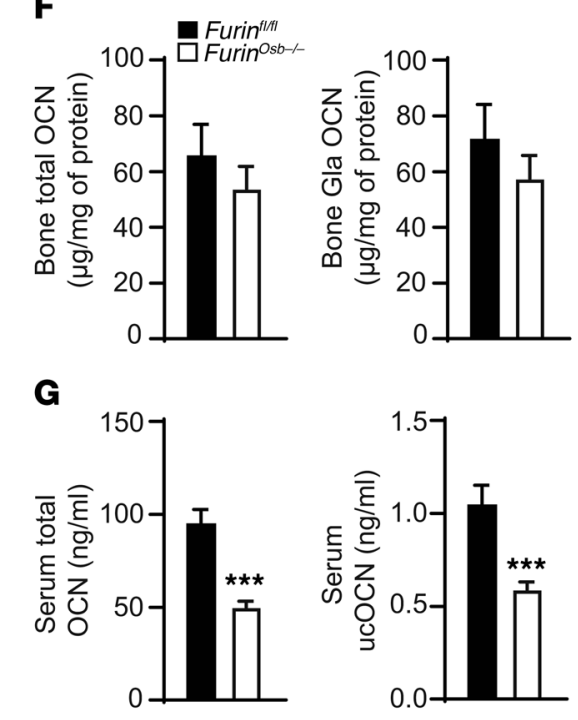

Figure 6. Impaired pro-OCN processing in Furin osb-/- mice. (A) Detection of the deleted allele ( $\mathrm{PCR}$ ) of Furin by PCR on genomic DNA extracted from different tissues. Flox PCR was used as a loading control. WAT, white adipose tissue; BAT, brown adipose tissue. (B) Furin expression in bone marrow-derived osteoblasts from Furin ${ }^{\text {fl/fl}}$ or Furin ${ }^{\text {osb- }-1-}$ mice assessed by Western blotting. (C) Quantification of furin protein levels relative to $\beta$-actin expressed as a percentage of Furin fl/fl. (D) Western blot analysis of total OCN and $\gamma$-carboxylated OCN in bone extracts from 9-month-old Furin ${ }^{f / f l}$ and Furin osb-/- mice. (E) Western blot analysis of OCN immunoprecipitated from the serum of Furin ${ }^{f / f f}$ or Furin ${ }^{o s b-/-}$ mice. (F) Total and $\gamma$-carboxylated OCN ELISA measurements in bone homogenates from 9-month-old Furin ${ }^{f / f l}(n=7)$ and Furinosb-/- $(n=8)$ mice. (C) Total and ucOCN ELISA measurements in serum from 9-month-old Furin ${ }^{f / f f l}$ $(n=16)$ and Furin ${ }^{\text {osb-/ }}(n=17)$ mice. The ELISAs used in $\mathbf{F}$ and $\mathbf{G}$ quantify both pro-OCN and mature OCN. Results indicate the mean \pm SEM. ${ }^{* *} P<0.01$ and ${ }^{* * *} P<0.001$, by unpaired, 2-tailed Student's $t$ test.

Furin $^{\text {osb-/ }}$ mice compared with levels in their control littermates (Supplemental Figure 1G), suggesting that the absence of furin in osteoblasts does not overtly impact osteoclast differentiation.

Previous studies have shown that the acidic $\mathrm{pH}$ generated during the process of bone resorption is responsible for OCN decarboxylation (13). Hence, we compared the decarboxylation of mature $\mathrm{OCN}$ and pro-OCN following incubation at a $\mathrm{pH}$ comparable to that found in the osteoclast resorption lacuna (i.e., $\mathrm{pH}$ 4.3). While the OCN contained in control mouse bone extracts was efficiently decarboxylated at $\mathrm{pH} 4.3$, the pro-OCN contained in Furin $^{\text {osb } / /}$ mouse bone extracts was not (Figure 7D and Supplemental Figure 1H). Importantly, anti-Gla OCN and anti-total OCN antibodies could detect mature $\mathrm{OCN}$ in control bone extracts, pro-OCN in the Furin ${ }^{\text {ssb- }}$ - mouse bone extracts, and mature OCN generated following the addition of recombinant furin to the latter extracts (Supplemental Figure 1I). Collectively, these results suggest that, in the absence of furin, osteoblasts secrete $\gamma$-carboxylated pro-OCN, which, like mature $\gamma$-carboxylated OCN, accumulates in the bone ECM. However, pro-OCN is poorly decarboxylated and released during bone resorption, resulting in reduced circulating levels of ucOCN.
Furin in osteoblasts is required to maintain glucose and energy homeostasis. To determine how the absence of pro-OCN cleavage impacts OCN endocrine functions, we next studied glucose metab-

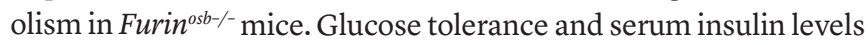
were normal in Furin ${ }^{\text {ssb- }- \text { mice }}$ at 3 and 6 months of age (Supplemental Figure 2, A-E). However, at 6 and 9 months of age, there was a significant increase in blood glucose levels under fed conditions in Furin ${ }^{\text {ss- }-/}$ mice compared with levels in littermate controls (Supplemental Figure 2F and Figure 8A). In addition, at 9 months

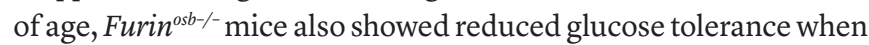
challenged with a glucose load (Figure 8B). Furthermore, in agreement with the fact that ucOCN normally favors insulin secretion by $\beta$ cells, serum insulin levels were significantly reduced in the fed state in Furin ${ }^{\text {sb- }-/}$ mice compared with levels in control littermates, while fasting levels were not affected (Figure 8C). These results are consistent with higher blood glucose levels in Furin ${ }^{\text {ssb-/ }}$ mice only in fed conditions or during a glucose tolerance test (GTT) (Figure 8, A and B). Moreover, and in line with the previously reported positive effect of ucOCN on insulin synthesis by $\beta$ cells, pancreatic insulin content was also reduced in Furin ${ }^{\text {ssb }-/}$ mice compared with that 
A

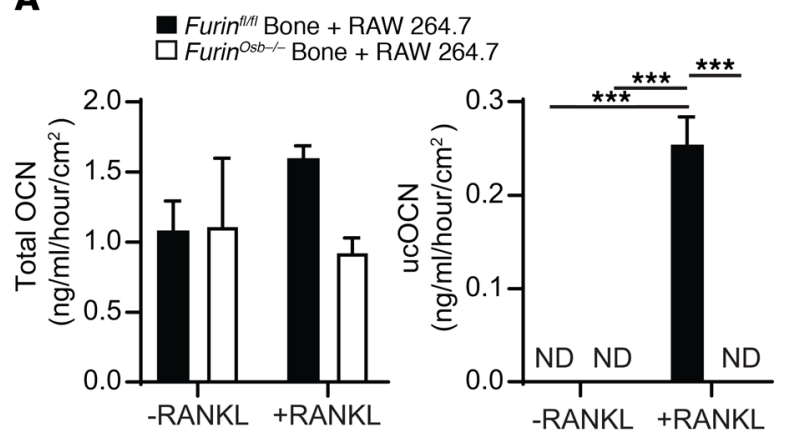

B

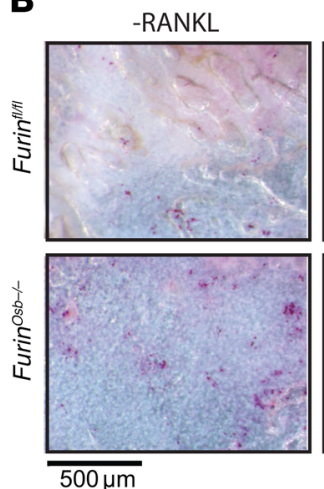

+ RANKL

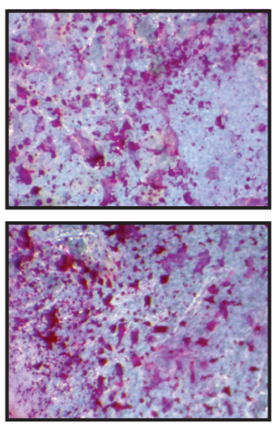

C

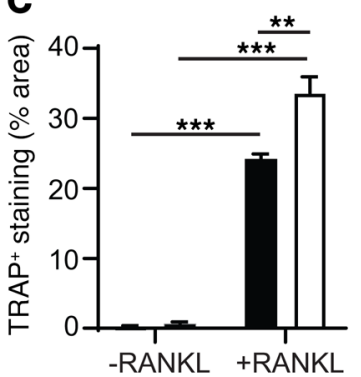

D

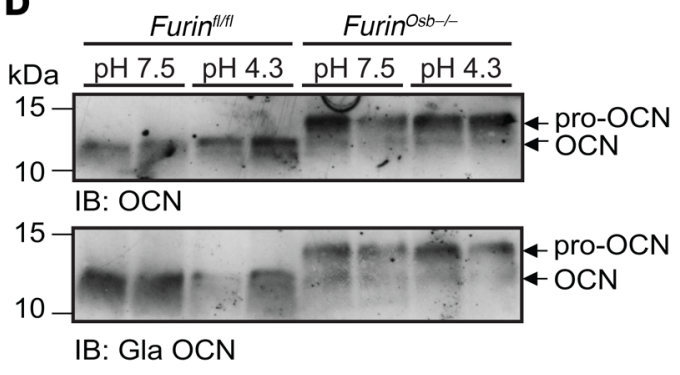

Furin ${ }^{\text {tifl }}$ Bone extracts $\square$ Furin $^{\mathrm{O} b-/-}$ Bone extracts

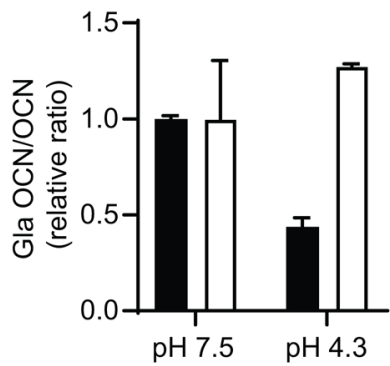

Figure 7. Pro-OCN is not efficiently decarboxylated by osteoclasts. (A and $\mathbf{B}$ ) In vitro resorption assay of devitalized calvaria from Furin ${ }^{f / f l}$ mice ( $n=3$ ) and Furin ${ }^{o s b-/-}$ mice $(n=3)$ in the presence of osteoclast-like RAW 246.7 cells, with or without 10 ng/mI RANKL. (A) Total OCN and ucOCN ELISA measurements in the cell culture supernatant. ND, not detected. (B) Representative image of TRAP staining of calvaria. Scale bar: $500 \mu \mathrm{m}$. (C) Percentage of area with TRAP-positive staining $(n=3)$. (D) Western blot analysis of bone extracts from Furif ${ }^{f l / f l}$ and Furin ${ }^{o s b-/-}$ mice incubated in phosphate-buffered solution at pH 7.5 or $\mathrm{pH} 4.3$ for 14 days at $37^{\circ} \mathrm{C}$. Graph shows quantification of the Gla OCN/OCN ratio (representative result from 3 independent experiments). The ELISAs used in A quantify both pro-OCN and mature OCN. Results represent the mean \pm SEM. ${ }^{* *} P<0.01$ and ${ }^{* * *} P<0.001$, by 1 -way ANOVA followed by Bonferroni's multiple comparisons test.

detected in control mice (Figure $8 \mathrm{D})$. Insulin sensitivity remained normal in Furin ${ }^{o s b-1-}$ mice at 3, 6, and 9 months of age (Supplemental Figure 2, G-I). Finally, challenging 4-week-old Furin ${ }^{\text {osb-/- }}$ mice with a high-fat, high-sucrose diet for 10 weeks was sufficient to reduce glucose tolerance in younger animals (Figure $8 \mathrm{E}$ ).

Since OCN also increases energy expenditure, we assessed the energy balance in control and Furin ${ }^{o s b-/}$ mice through indirect calorimetry. At 3 and 6 months of age, chow-fed Furin ${ }^{\text {osb-/- }}$ mice displayed reduced $\mathrm{O}_{2}$ consumption and $\mathrm{CO}_{2}$ production in the dark phase, i.e., when the mice were more active (Figure 9, A and $\mathrm{B}$, and Supplemental Figure 2, J and $\mathrm{K}$ ), which resulted in a significant decrease in global energy expenditure (Figure 9C and Supplemental Figure 2L). We observed no decrease in physical activity in Furin ${ }^{o s b-/-}$ mice during the dark and light phases (Figure 9, D and E), suggesting that the reduced energy expenditure of the Furin ${ }^{o s b-/}$ mice was not caused by a diminution in the voluntary movements of these animals. The lower energy expenditure in Furin ${ }^{\text {osb-/- }}$ mice was associated with an increased epididymal fat pad weight and whole-body fat tissue percentage at 12 months of age compared with control mice, although their body weight was not significantly changed compared with that of their littermate controls, whether the mice were fed a normal chow diet or a highfat diet (Figure 9F and Supplemental Figure 3, A-D). Overall, the phenotypic characterization of Furin ${ }^{o s b-/-}$ mice revealed that furin, through its expression in osteoblasts, plays a role in regulating glucose and energy metabolism. Our findings also support the con- clusion that pro-OCN processing by furin is required for the complete activation of this hormone.

Furin regulates appetite through its expression in osteoblasts. Furin $^{\text {osb-/- }}$ mice are characterized by normal glucose tolerance at 3 and 6 months of age when fed a regular chow diet (Supplemental Figure 2, A and D). Moreover, insulin sensitivity, as assessed by insulin tolerance tests (ITTs), was normal in Furin ${ }^{o s b-/-}$ mice in all the age groups tested (Supplemental Figure 2, G-I). This contrasts with Ocn inactivation that results in both glucose intolerance and insulin resistance as early as 3 months of age in mice fed a chow diet (2). Likewise, on a chow diet, Furin ${ }^{o s b-/}$ mice had lower fat accumulation than did $\mathrm{Ocn}^{-/}$mice (Figure 9F and ref. 2). Hence, the metabolic phenotypes are delayed or milder in Furin ${ }^{o s b-/-}$ mice compared with $\mathrm{Ocn}^{-/-}$mice.

Although several possibilities could explain these differences, one of them is that furin negatively modulates other aspects of energy metabolism independently of OCN. In support of this hypothesis, we observed a significant reduction of approximately

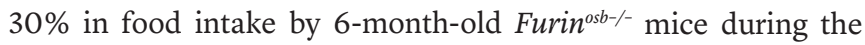
dark phase (Figure 9G). Accordingly, cumulative food intake was reduced by approximately $20 \%$ over a 3 -day period (Figure $9 \mathrm{H}$ ). We found that cumulative food intake was also reduced by approximately $15 \%$ in 3-month-old Furin ${ }^{\text {osb-- }}$ mice (Supplemental Figure $3, \mathrm{E}$ and $\mathrm{F}$ ). In contrast, $\mathrm{Ocn}^{-/-}$animals of the same age (6 months) and genetic background (i.e., C57BL/6J) had normal food intake compared with their WT littermates (Figure 9, I and J). Circulating 
A

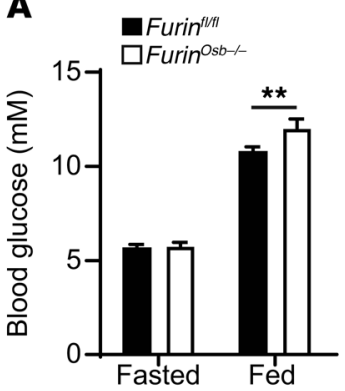

B

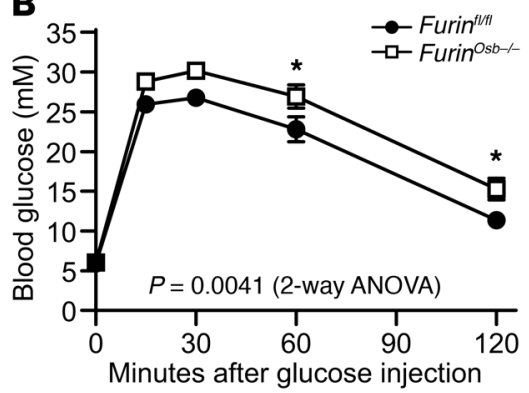

C

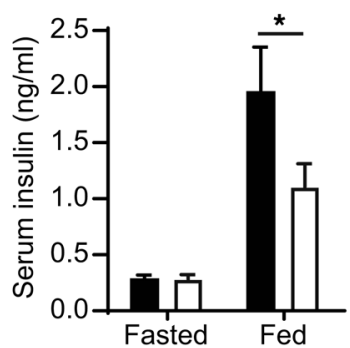

D

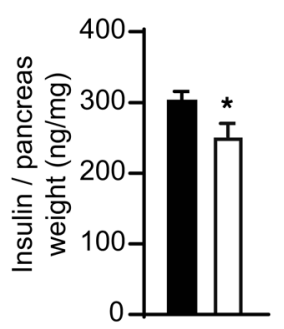

E

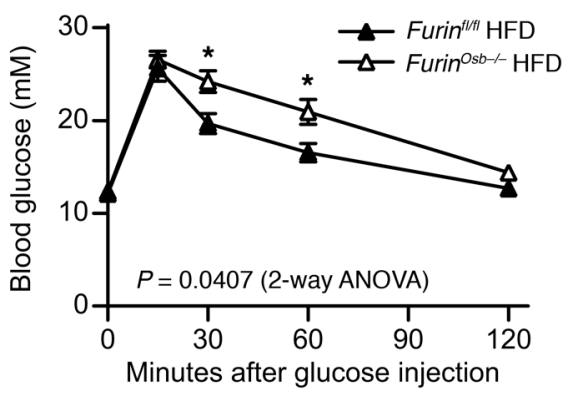

Figure 8. Reduced glucose tolerance in Furin ${ }^{\text {osb-/- }}$ mice. (A) Fasting and fed blood glucose levels in 9-month-old Furin ${ }^{f l / f l}(n=12)$ and Furin ${ }^{o s b-/-}(n=10)$ mice. (B) GTT results for 9-month-old Furin fl/fl $(n=21)$ and Furin ${ }^{\text {osb-/- }}(n=16)$ mice. Mice were fasted for 16 hours and injected i.p. with $2 \mathrm{~g} / \mathrm{kg}$ glucose. (C) Fasting and fed serum insulin levels in 9-month-old Furin ${ }^{f / f l}(n=12)$ and Furin ${ }^{\text {osb- }- \text { - }}$ $(n=10)$ mice. (D) Pancreas insulin content in 9-month-old Furin ${ }^{f l / f l}(n=10)$ and Furin ${ }^{\text {osb-/- }}(n=10)$ mice. (E) GTT results for 4-month-old Furin ${ }^{f l / f l}(n=8)$ and Furin ${ }^{\text {osb-/- }}(n=7)$ mice after 10 weeks on a high-fat, high-sucrose diet (HFD). Mice were fasted for 6 hours and then injected i.p. with $1 \mathrm{~g} / \mathrm{kg}$ glucose. Metabolic assessments were performed on male mice fed a normal chow diet (A-D) or a HFD (E). Results represent the mean \pm SEM. ${ }^{*} P<0.05$ and ${ }^{* *} P<0.01$, by 2-way ANOVA for repeated measurements with Bonferroni's multiple comparisons test ( $\mathbf{A}-\mathbf{C}$ and $\mathbf{E}$ ) or by unpaired, 2-tailed Student's $t$ test (D). leptin levels were not changed in Furin ${ }^{o s b-/-}$ mice (Supplemental Figure 3G). Collectively, these observations suggest that Furin ${ }^{o b-1-}$ mice do not develop insulin resistance as robustly as do $\mathrm{OCn}^{-1-}$ mice, in part because of a reduction in calorie intake, a condition shown previously to improve insulin sensitivity in mice (36).

To test this possibility directly, we pair fed 4-month-old control and Furin ${ }^{\text {ssb-/- }}$ mice for 2 to 4 weeks to ensure similar calorie intake in both groups (Supplemental Figure 3H) before assessing their glucose tolerance and insulin sensitivity. As shown in Figure $10 \mathrm{~A}$, Furin ${ }^{\text {osb- }-1}$ mice were significantly heavier than the pair-fed control mice, and this was associated with an increase in the percentage of whole-body fat tissue (Figure 10B). When pair fed with their control littermates, Furin ${ }^{o s b-/-}$ mice showed higher feeding blood glucose levels and a glucose intolerance phenotype (Figure 10, C and D). Finally, under these conditions, the Furin ${ }^{o s b-/-}$ mice were found to be insulin resistant in comparison with the control mice, as assessed by an ITT (Figure 10E). Importantly, most of these phenotypes were not observed at the same age when control and Furin ${ }^{o s b-1-}$ mice were fed ad libitum (Supplemental Figures 2 and 3). Since LCN2 was recently identified as a bone-derived hormone regulating appetite (37), we tested whether furin regulates appetite by processing LCN2. However, we found that furin was unable to cleave recombinant LCN2 in vitro and that the apparent molecular weight of LCN2 on Western blotting was not changed in bone extracts from Furin ${ }^{o s b-/}$ mice compared with that observed in control bone extracts (Supplemental Figure 4, A and B). Finally, following refeeding, we found that circulating levels of LCN2 were the same in control and Furin ${ }^{o s b-/-}$ mice (Supplemental Figure 4C). Together, these results support the notion that inactivation of furin in osteoblasts produces two distinct effects on energy metabolism: it reduces energy expenditure, glucose tolerance, and insulin sensitivity on one hand, and it decreases appetite on the other hand. The first effect is most likely OCN dependent, while the second one occurs through an OCN- and LCN2-independent mechanism.

\section{Discussion}

In this study, we identified furin as the PC responsible for the conversion of pro-OCN to mature OCN in osteoblasts. Our results show that pro-OCN processing regulates the circulating levels of active unOCN, since pro-OCN is less efficiently released and decarboxylated from the bone ECM during the process of bone resorption. Accordingly, mice in which the Furin gene is inactivated in osteoblasts only are characterized by metabolic anomalies similar to those reported in Ocn-deficient animals. Together, we believe this work identifies a previously undescribed regulatory mechanism of bone endocrine function and shows that OCN activation, as with several other peptide hormones, is dependent on the activity of a specific PC.

Pro-OCN processing is dependent on furin in osteoblasts. With the use of a variety of approaches, we established that, in osteoblasts, pro-OCN maturation is exclusively performed by furin and not by the other members of the PC family. First, in vitro assays showed that pro-OCN is most efficiently processed by furin compared with the 3 other PCs expressed by osteoblasts. Second, inactivation of Furin in cultured osteoblasts completely abrogates pro-OCN processing. Third, in mice lacking Furin only in osteoblasts, pro-OCN failed to be processed and accumulated in the bone ECM. PC5 is another PC that is required for normal patterning of the axial skeleton through the processing of progrowth differentiation factor 11 (pro-GDF11) (35). PC5 has been reported to be expressed in osteoblasts and osteocytes, where it may process FGF23, another bone-derived hormone regulating phosphate homeostasis (34). However, our data show that, although PC5A is expressed in primary osteoblasts and cleaves pro-OCN in vitro, the inactivation in this cell type of Pcsk5, encoding PC5A and PC5B isoforms, does not affect pro-OCN processing in cell culture or in vivo. PC5A and pro-OCN may not colocalize to the same cellular compartment, as PC5A is activated only after it reaches the cell surface (18).

Since furin deficiency leads to lethality between E10.5 and E11.5, this PC has been implicated in a limited number of 
A
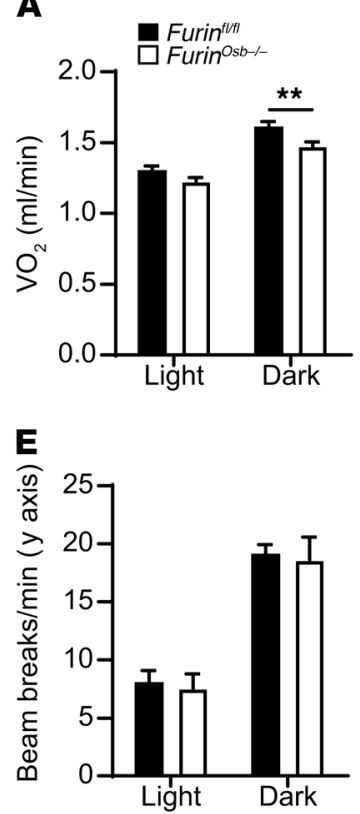

I

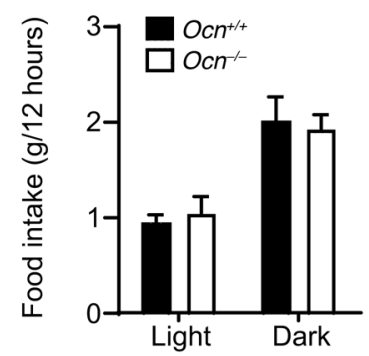

B
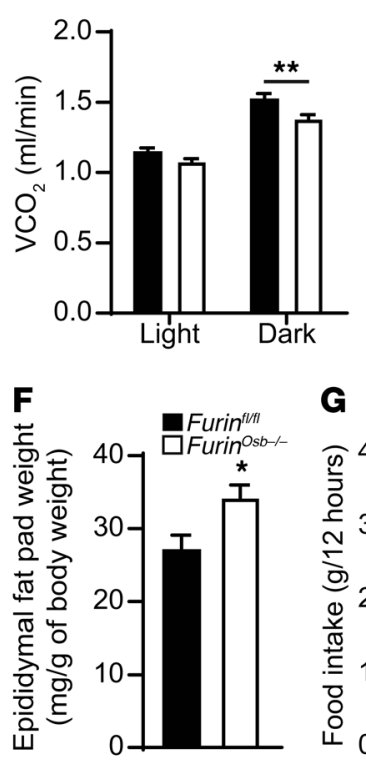

J

G
C

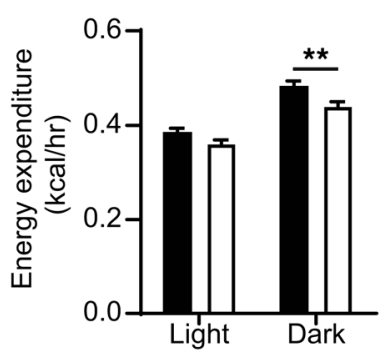

D

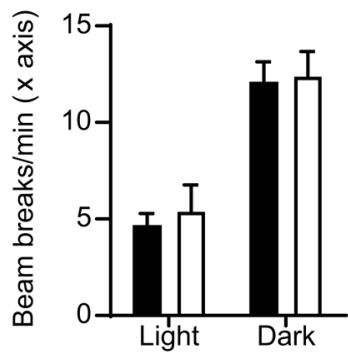

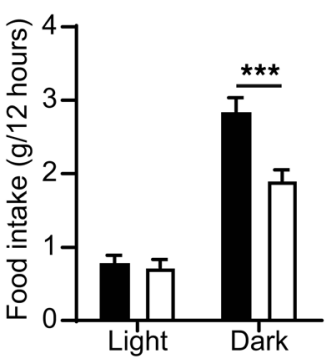

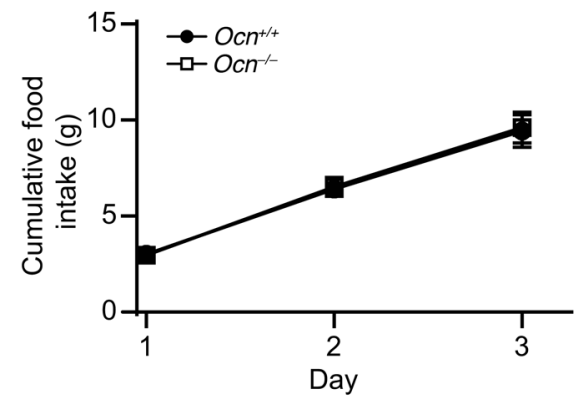

H

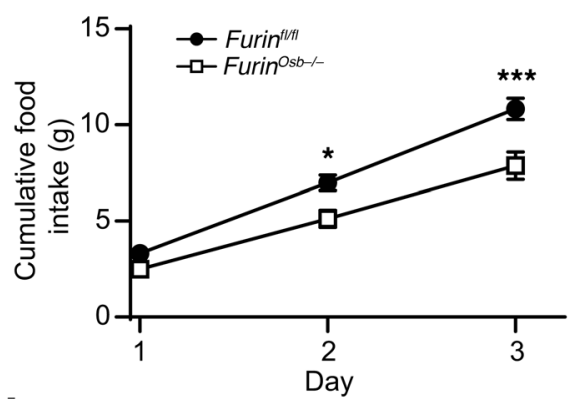

Day

Figure 9. Reduced energy expenditure and food intake in Furin ${ }^{o s b-/-}$ mice. (A-E) Metabolic parameters of 6-month-old Furin ${ }^{f / f l}(n=11)$ and Furin ${ }^{o s b-/-}$ $(n=9)$ mice. $\mathrm{O}_{2}$ consumption $(\mathbf{A}), \mathrm{CO}_{2}$ release $(\mathbf{B})$, heat production (energy expenditure) (C), and activity on the $x$ axis $(\mathbf{D})$ and $y$ axis $(\mathbf{E})$. (F) Epididymal fat pad weight normalized to the body weights of 12-month-old Furin ${ }^{f l / f l}(n=12)$ and Furin ${ }^{o s b-/-}(n=12)$ mice fed a normal chow diet. (G-J) Food intake by 6-monthold Furin ${ }^{f / f l}(n=11)$, Furin $n^{\text {osb-/- }}(n=9), \mathrm{Ccn}^{+/+}(n=8)$, and $\mathrm{Ccn}^{-/-}(n=8)$ mice. (C and I) Food intake during the light and dark periods. (H and J) Cumulative food intake over a 3-day period. Results represent the mean $\pm \mathrm{SEM}$. ${ }^{*} P<0.05$, ${ }^{* *} P<0.01$, and ${ }^{* * *} P<0.001$, by 2-way ANOVA for repeated measurements with Bonferroni's multiple comparisons test (A-E and $\mathbf{G}-\mathbf{J})$ or by unpaired, 2-tailed Student's $t$ test (F).

postnatal functions so far. Furin was shown to be essential for the maintenance of peripheral immune tolerance through its expression in $\mathrm{T}$ lymphocytes (24), to play a role in the granular acidification of pancreatic $\beta$ cells (23), and to be required for cardiac development through its expression in endothelial cells (38). In contrast, studies of furin functions in liver showed that it might act redundantly with other PCs in this tissue $(22,30)$. In the present study, we show that furin is the cognate PC for proOCN activation in osteoblasts, providing genuine in vivo evidence for a nonredundant role of furin in this cell type. In classical endocrine cells, prohormones (e.g., proglucagon, proinsulin, and pro-opiomelanocortin) are generally converted to active hormones by either PC1 and/or PC2 $(39,40)$. Consistent with the notion that the osteoblast is a nonclassical endocrine cell, our data show that both PC1 and PC2 are not expressed in osteoblasts and that instead furin is responsible for pro-OCN conversion. Consequently, although furin was previously shown in cell culture to be responsible for proparathyroid hormone (pro-
PTH) conversion (41) and in the stepwise generation of growth hormone-releasing hormone (GHRH) together with PC1 (42), our study is the first to our knowledge to reveal the sole requirement for furin in the activation of a specific peptide hormone and to imply that furin has a role in endocrine regulation in vivo.

Processing of OCN is not dependent on its $\gamma$-carboxylation. The precursors of other $\gamma$-carboxylated proteins, such as thrombin and factor IX, also contain a propeptide that consists of a recognition signal for the $\gamma$-glutamyl carboxylase as well as a dibasic motif (RR) in their C-terminus (15). It is thought that processing of the propeptide in $\gamma$-carboxylated proteins could depend on their efficient $\gamma$-carboxylation (43). Although the OCN propeptide appears to contain a putative $\gamma$-glutamyl carboxylase recognition motif (44), mature OCN can be $\gamma$-carboxylated in vitro in the absence of its propeptide (16). Through several approaches, including mass spectrometry, we showed that preventing pro-OCN $\gamma$-carboxylation, either pharmacologically or genetically, did not affect its processing by furin, thereby establishing that these 2 posttranslational 
A
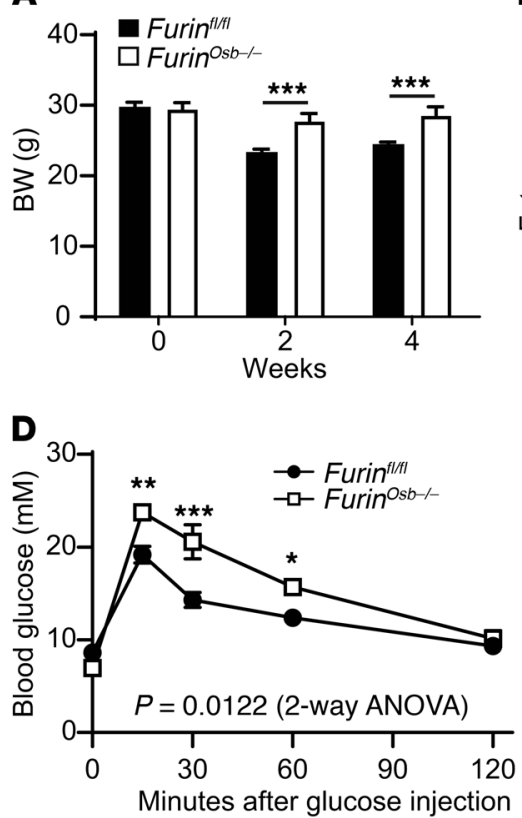

B

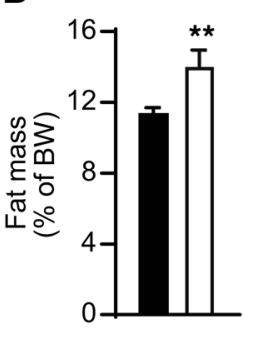

C
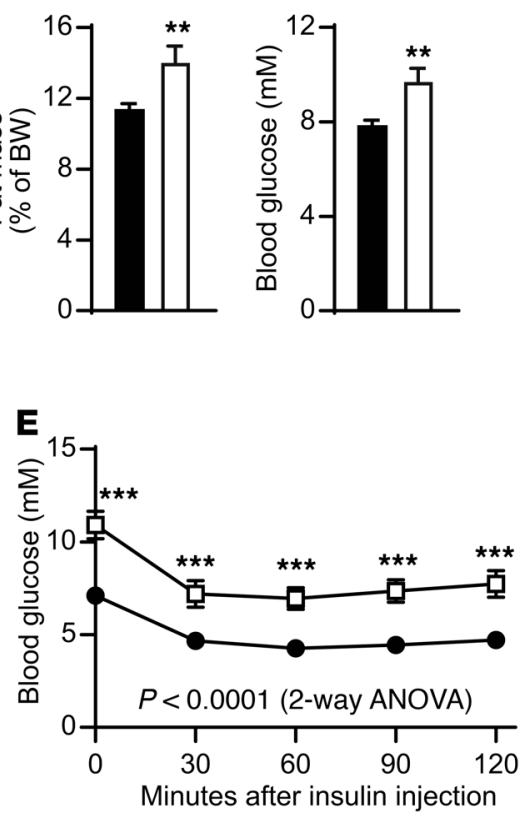

Figure 10. Pair feeding reveals a more severe metabolic phenotype in Furin osb-/- mice. Metabolic phenotyping of 4-month-old pair-fed Furin fl/fl $(n=12)$ and Furinosb-/$(n=7)$ mice. (A) Body weight before initiation of pair feeding (week 0 ) and after 2 and 4 weeks of pair feeding. (B) Percentage of total fat mass over body weight after 4 weeks of pair feeding. (C) Fed blood glucose measurement after 3 weeks of pair feeding. (D) GTT results after 4 weeks of pair feeding. Mice were fasted for 16 hours and injected i.p. with $2 \mathrm{~g} / \mathrm{kg}$ glucose. (E) ITT results after 4 weeks of pair feeding. Mice were fasted for 5 hours and injected i.p. with $0.3 \mathrm{U} / \mathrm{kg}$ of insulin. Results represent the mean \pm SEM. ${ }^{*} P<0.05$, ${ }^{* *} P<0.01$, and ${ }^{* * *} P<0.001$, by 2-way ANOVA for repeated measurements with Bonferroni's multiple comparisons test (A, $\mathbf{D}$, and $\mathbf{E}$ ) or by unpaired, 2-tailed Student's $t$ test (B and C). modifications occur independently of each other. In line with our findings, earlier studies reported that inhibition of $\gamma$-carboxylation using warfarin in the ROS 17/2.8 rat osteosarcoma cell line caused the intracellular accumulation of pro-OCN, but also the release of mature decarboxylated $\operatorname{OCN}(45,46)$.

Furin in osteoblasts regulates whole-body energy metabolism in part through OCN. Beyond 9 months of age, Furin ${ }^{o s b-/-}$ mice display reduced glucose tolerance, decreased serum insulin levels, lower energy expenditure, and increased white adipose tissue mass compared with their control littermates. These phenotypes are consistent with those of $\mathrm{Ocn}^{-/}$mice and support the conclusion that pro-OCN processing by furin is required for the activation of this hormone. Nonetheless, some metabolic anomalies in Furin $^{\text {osb-/- }}$ mice were delayed or not detected (e.g., insulin resistance) as compared with Ocn-deficient mice (2).

Furin $^{o s b-/-}$ mice also exhibit reduced food intake, which was not observed in $\mathrm{Ocn}^{-/-}$mice. Because reduced calorie intake was shown to improve insulin sensitivity (36), this could explain why glucose intolerance and insulin sensitivity phenotypes are milder in Furin $^{o s b-/-}$ mice than in $\mathrm{Ocn}^{-/-}$mice. Accordingly, pair feeding revealed a more severe glucose intolerance and insulin sensitivity phenotype in the Furin ${ }^{o s b-/}$ mice. Interestingly, although OCN appears to be a major mediator of bone endocrine action on energy metabolism, recent findings suggest the existence of additional bone-derived factor(s) implicated in the control of glucose metabolism. For instance, partial ablation of osteoblasts in adult mice resulted in reduced glucose tolerance, hypoinsulinemia, and decreased insulin sensitivity, as observed in $\mathrm{Ocn}^{-/-}$mice (47). However, unlike OCN deficiency, osteoblast ablation also results in increased food intake. This earlier work supports the existence of at least 1 additional osteoblast-derived factor negatively regulating appetite and insulin sensitivity. Whether such an osteoblast-derived factor could be processed and possibly inactivated by furin is a possibility with regard to the reduced food intake detected in the Furin ${ }^{o s b-/-}$ mice. A recent study identified LCN2 as an osteoblast-derived hormone that regulates appetite (37), however, our data suggest that furin does not modulate LCN2 processing or serum levels. Hence, further work will be required to identify the additional osteoblast-derived factor involved in appetite control and to determine how furin regulates its function.

Pro-OCN is less efficiently released during the process of bone resorption. Our cellular and in vivo experiments establish that, in the absence of furin, pro-OCN is not converted to mature OCN, but is still efficiently $\gamma$-carboxylated and secreted. These observations suggest that pro-OCN processing is not required for OCN to be secreted. Similarly, it was previously shown that profactor IX, which can be processed in vitro by furin, is normally secreted in its $\gamma$-carboxylated proform in heterologous cells. However, in contrast to mature factor IX, profactor IX was found to be unable to bind phospholipid vesicles in the presence of $\mathrm{Ca}^{2+}(48)$. We have previously demonstrated that $\gamma$-carboxylation of OCN is absolutely required for its binding to bone ECM, since in mice lacking $G g c x$ in osteoblasts, bones are depleted of OCN (12). In contrast, pro-OCN does accumulate normally in the bones of Furin ${ }^{\text {osb-r- }}$ mice, suggesting that the propeptide does not prevent binding of the OCN Gla residues to the mineral component of bone ECM. Hence, retention of the propeptide has different effects on the calcium-binding properties of pro-OCN and profactor IX.

Although bone OCN content was normal in Furin ${ }^{o s b-/}$ mice, the circulating levels of total and ucOCN were reduced. $\gamma$-carboxylated OCN was shown to be partially decarboxylated and activated during the process of bone resorption in a nonenzymatic reaction requiring the acidic $\mathrm{pH}$ of the resorption lacuna generated by the osteoclasts $(13,14)$. Other data presented here show that pro-OCN is less efficiently decarboxylated than is mature OCN during bone resorption and following incubation at $\mathrm{pH} 4.3$, suggesting that the retention of the propeptide protects OCN Gla residues from the effect of the low $\mathrm{pH}$ generated by osteoclasts. 
What is the role of OCN propeptide if it is not required for OCN $\gamma$-carboxylation? The propeptide domain might facilitate the entry of the 96-aa-long pre-pro-OCN in the ER, its cleavage by the signal peptidase, the exit of the 72-aa-long pro-OCN from the ER, and its transport through the secretory pathway until it reaches the TGN, where furin is active (26). In support of this notion, expression of OCN without its propeptide sequence in osteoblasts greatly reduces the amount of mature OCN secreted in the media (El-Rifai and Ferron, unpublished observations). Finally, it was reported that a polypeptide must have a minimum size of at least 50 aa in order to be recognized by the signal recognition particle and threaded through the membrane of the ER (49), making the 46-aa-long OCN too small to be efficiently secreted on its own.

The lumen of the TGN is characterized by an acidic $\mathrm{pH}$, i.e., as low as pH 5.9 in HeLa cells (50). Interestingly, consistent with its preferential action in the TGN, furin is more active in vitro at $\mathrm{pH}$ 6 than at $\mathrm{pH} 7.5$ (51). Hence, we can also speculate that the normal function of OCN propeptide could be to protect OCN from undergoing premature decarboxylation while it is transiting through the TGN and the constitutive secretory pathway. Alternatively, the presence of the propeptide may prevent OCN from premature folding in the TGN, since it was reported that OCN can fold in the absence of calcium ions at pH 6 and below (52). Further investigation will be needed to specifically address these possibilities.

In summary, this work provides in vitro and in vivo evidences that the processing of the OCN prohormone by furin regulates the activation and endocrine function of OCN. Moreover, we established that furin in osteoblasts plays an important role in the regulation of energy metabolism by bone through OCN-dependent and -independent mechanisms.

\section{Methods}

Animal models. The Furin ${ }^{f / f l}$ mice were generated by introducing loxP sites upstream and downstream of exon 2 of the Furin gene (30). $G g c x^{f / f l}$ mice were generated by introducing a loxP site upstream of exon 1 and a loxP site downstream of exon 2 of the $G g c x$ gene (12). Pcsk $5^{f / f l}$ mice were generated by introducing loxP sites on each side of Pcsk5 exon 1 (35). Each of these strains was crossed with $\mathrm{OCN}$-Cre-transgenic mice, which express Cre recombinase under the control of a fragment of the human OCN promoter (31) and which were previously shown not to display any metabolic anomalies, in order to generate, respectively, Furin ${ }^{\text {fl/ft }}$ OCN-Cre (Furin $\left.{ }^{o s b-/}\right), G g C x^{f / f l} O C N-C r e\left(G g C x^{o s b-/}\right)$, and Pcsk5 $5^{f / f l} O C N-C r e$ (Pcsk5 $\left.5^{o b-/-}\right)$ mice. Ocn ${ }^{-/}$mice were generated by replacing Ocn1 (Bglap1) and Ocn2 (Bglap2) genes in the mouse Ocn cluster with a neomycin resistance cassette through homologous recombination (53). All strains used were backcrossed on a C57BL/6J genetic background more than 10 times and maintained in an IRCM specific pathogen-free animal facility (SPF) under 12-hour dark/12-hour light cycles. Male mice fed a normal chow diet were used in all experiments, unless otherwise noted. Tissue DNA was extracted by standard procedure, and PCR was performed with the genotyping PCR primers listed in Supplemental Table 1.

Metabolic analysis. For GTTs, adult male mice fed a normal chow diet were fasted overnight for 16 hours. Blood glucose levels were measured after fasting and at 15, 30, 60, and 120 minutes following i.p. injection with $2 \mathrm{~g} / \mathrm{kg}$ glucose. In some experiments, mice were fed a 60\% high-fat diet (D12331-5I; Research Diets) for 10 weeks starting from 4 weeks of age. In this case, mice were fasted for 6 hours and injected i.p. with $1 \mathrm{~g} / \mathrm{kg}$ glucose for the GTT. To measure pancreatic insulin content, pancreas was collected, and insulin was extracted in acid-ethanol as previously described (13). Serum insulin and pancreatic insulin levels were assessed using a commercial insulin ELISA (Mercodia). Serum leptin and LCN2 levels were measured using a mouse leptin ELISA kit (EZML-82K; EMD Millipore) and a mouse lipocalin-2 ELISA kit (MLCN20; R\&D Systems), respectively. In the pair-feeding experiment, each mouse was housed in a separate cage. Furin ${ }^{\text {osb }-/}$ mice were fed a normal chow diet ad libitum, and food intake was measured and averaged daily. The corresponding amount of food was given to the Furin $^{f / f l}$ mice. Metabolic tests were performed between the third and fourth weeks of pair feeding.

Mouse $\mathrm{O}_{2}$ consumption, $\mathrm{CO}_{2}$ release, activity, and food intake were assessed using an 8-chamber Promethion Continuous Metabolic System (Sable Systems International). After a 48-hour acclimation period, $\mathrm{O}_{2}$ consumption $\left(\mathrm{VO}_{2}\right)$ and $\mathrm{CO}_{2}$ production $\left(\mathrm{VCO}_{2}\right)$ data were collected for 72 hours. Heat production was calculated by indirect calorimetry using the following formulas: heat in $\mathrm{kcal} / \mathrm{hr}=60 \times(0.003941$ $\times \mathrm{VO}_{2}+0.001106 \times \mathrm{VCO}_{2}$ ), where $\mathrm{VO}_{2}$ and $\mathrm{VCO}_{2}$ are in units of $\mathrm{ml} /$ min. Physical activity was measured using infrared beams connected to the system. Total body fat was measured using a body composition analyzer (echo-MRI) and normalized to body weight.

DNA construct, cell culture, and transfection. Mouse pro-OCN cDNA was cloned into a pIRES2-EGFP-V5 plasmid in EcoRI and AgeI restriction sites. Different OCN mutants were generated by sitedirected mutagenesis using specific primers (Supplemental Table 1). Primary osteoblasts or Chinese hamster ovary ldlD cells (CHO-ldlD; originating from the M. Krieger laboratory [ref. 54]) were transfected with jetPRIME Reagent (Polypus transfection). Following an overnight incubation, media were changed to secretion media (FBS-free $\alpha \mathrm{MEM}$ plus 2mM L-glutamine for osteoblasts or FBS-free DMEM/F12 for CHO-ldlD cells) supplemented with $22 \mu \mathrm{M}$ vitamin $\mathrm{K}_{1}$ (Sandoz). Twenty-four hours later, media were collected, and cells were lysed in protein lysis buffer (20 mM Tris-HC, pH 7.4, $150 \mathrm{mM} \mathrm{NaCl}, 1 \mathrm{mM}$ EDTA, 1 mM EGTA, 1\% Triton, $1 \mathrm{mM}$ PMSF, and $1 \times$ protease inhibitor cocktail) and analyzed by Western blotting. In some experiments, warfarin (50 $\mu \mathrm{M}$; Santa Cruz Biotechnology), D6R (20 $\mu \mathrm{M}$; Calbiochem), or Dec-RVKR-CMK (50 $\mu \mathrm{M}$; Tocris) was added to the secretion media.

Osteoblast preparation. Primary mouse osteoblasts were isolated from 3-day-old mouse calvariae through serial collagenase (Worthington Biochemical Corp.) digestions as previously described (12). To generate Furin $^{-/-}$or Pcsk5 $5^{-/-}$osteoblasts, Furin ${ }^{f / f l}$ or Pcsk $5^{\text {fl/f }}$ osteoblasts were infected with Cre-GFP-expressing adenovirus (University of Iowa) at 200 MOI per cell. Control osteoblasts were obtained by infecting Furin $^{f / f l}$ or Pcsk $5^{f / f l}$ osteoblasts with GFP-expressing adenovirus. Osteoblast differentiation was induced by supplementing osteoblast culture media ( $\alpha$ MEM plus $10 \%$ FBS and 2mM L-glutamine) with $5 \mathrm{mM} \beta$ glycerophosphate and $100 \mu \mathrm{g} / \mathrm{ml} \mathrm{L}$-ascorbic acid for 21 days. Bone marrow-derived osteoblasts were prepared as previously described (12). Briefly, long bones of 3 week-old-mice were dissected, and bone marrow cells were flushed with $\alpha$ MEM. Cells were then plated in 6-well plates at a density of $5 \times 10^{6}$ cells per well. After 7 days, media were changed to differentiation media (osteoblast media supplemented with $100 \mu \mathrm{g} / \mathrm{ml} \mathrm{L}$-ascorbic acid, $5 \mathrm{mM} \beta$-glycerophosphate, and $10^{-8} \mathrm{M}$ dexamethasone). After 14 days of differentiation, cells were used for mRNA or protein analysis.

Biochemical studies. For OCN immunoprecipitation, Furin $n^{f / f l}$ or Furin $^{\text {osb-/ }}$ serum was diluted 1:50 with $1 \times$ PBS containing $1 \mathrm{mM}$ PMSF, 
and $1 \times$ protease inhibitor cocktail and incubated overnight at $4^{\circ} \mathrm{C}$ in the presence of the OCN antibodies indicated in Figure 5D, Figure 6E, and in Supplemental Figure 1F. Protein G agarose beads prewashed with $1 \times$ PBS were added and incubated for 4 hours at $4^{\circ} \mathrm{C}$. Beads were spun down and washed 4 times with $1 \times$ PBS before Western blot analysis. Proteins were resolved on $15 \%$ Tris-tricine SDS-PAGE gel for OCN detection, whereas $7.5 \%$ Tris-glycine gel was used for the detection of furin and the insulin receptor. The antibodies used in this study were: anti-OCN (total) polyclonal goat antibody, which recognize aa 26-46 corresponding to mature mouse OCN (55); anti-Gla OCN polyclonal goat antibody, which recognize Gla13 OCN aa 11-26 corresponding to carboxylated mature OCN (55); anti- $\beta$-actin (mouse, clone AC-15, A5441; Sigma-Aldrich); anti-V5 (mouse, clone V5-10, V8012; Sigma-Aldrich); anti-furin (rabbit, PA1-062; Thermo Fisher Scientific); anti-insulin receptor $\beta$ (mouse, clone L55B10, 3020; New England BioLabs); and anti-LCN2 (goat, AF1857; R\&D Systems). Serum and cell supernatant levels of carboxylated, undercarboxylated, and total OCN were measured using ELISAs as described previously (55). Briefly, the Gla ELISA detects OCN only when it is carboxylated on the Glu13 residue. The Glu ELISA quantifies the uncarboxylated form of OCN. The total ELISA detects OCN, whether it is carboxylated or not. OCN measurements in bone homogenate were normalized to total protein content, as assessed by Bradford assay.

GST pro-OCN preparation and in vitro digestion assay. Mouse proOCN cDNA (WT or R48A/R49A, i.e., RR/AA mutant) was cloned into pGEX4T3 in BamHI and EcoRI restriction sites (Supplemental Table 1). Plasmids were transformed in Rosetta (DE3) pLys S competent bacteria (EMD Millipore), and recombinant GST proteins were purified using glutathione resin (Genscript). Recombinant full-length LCN2 protein was provided by S. Kousteni (Columbia University, New York, NY, USA). The cDNA of the soluble form of various PCs were cloned into the pIRES2-EGFP plasmid and transfected into HEK293 cells (ATCC) using Lipofectamine 2000 (Life Technologies, Thermo Fisher Scientific). Twenty-four hours after transfection, cells were allowed to secrete in serum-free media for twenty-four hours, and supernatant was harvested. PCs were concentrated using centrifugal filters (50kDa MWCO Amicon; EMD Millipore) and stored in 20\% glycerol at $-80^{\circ} \mathrm{C}$. Kexin-like protease activity was assessed by in vitro cleavage of $100 \mu \mathrm{M}$ Pyr-RTKR-AMC (Tocris) in PC buffer ( $25 \mathrm{mM}$ Tris- $\mathrm{HCl}$, $\mathrm{pH}$ 7.0, $1 \mathrm{mM} \mathrm{CaCl}, 1 \mathrm{mM} \beta$-mercaptoethanol). Soluble extracellular PACE4 expressed in HEK293 cells revealed no enzymatic activity (data not shown); hence, recombinant soluble PACE4 expressed and purified from insect cells (provided by R. Day, Université de Sherbrooke, Sherbrooke, Canada [ref. 21]) was used for the assay. Pro-GST-OCN or LCN2 in vitro digestions were performed in PC buffer with $200 \mathrm{ng}$ substrate and 0.7 units of PC, and mature OCN was detected by Western blotting using anti-OCN antibodies.

Gene expression. Osteoblasts and calvaria total RNA were isolated using TRIzol Reagent (Invitrogen, Thermo Fisher Scientific). RNAs were reverse transcribed using M-MLV Reverse Transcriptase (Invitrogen, Thermo Fisher Scientific), and real-time quantitative PCR (qPCR) was performed using SYBR Green qPCR Master Mix (BiMake) and gene-specific primers (see Supplemental Table 1). Expression levels were normalized to Actb expression levels.

Additional details on the methods used in this study are provided in the supplemental material.

Statistics. Statistical analyses were performed using GraphPad Prism software version 7.03. Results are shown as the mean \pm SEM. For single measurements, an unpaired, 2-tailed Student's $t$ test was used, while 1-way ANOVA followed by Bonferroni's post test was used for comparison of more than 2 groups. For repeated measurements (e.g., GTTs and ITTs), a repeated-measures 2-way ANOVA followed by Bonferroni's post test was used. A P value of less than 0.05 was considered statistically significant. All experiments were repeated at least 3 times or performed on at least 3 independent animals.

Study approval. All animal use complied with the guidelines of the Canadian Committee for Animal Protection and was approved by IRCM Animal Care Committee.

\section{Author contributions}

MF and JL designed experiments. OER performed most experiments, with crucial help from JC (genetic and pharmacological studies with primary osteoblasts), JL (metabolic cages and metabolic tests), CJ (in vitro PC assay), DF (LC-MS/MS analysis), and DSR (PC activity). RE and NGS provided $P c s k 5^{f / f l}$ mice and plasmids. JWC provided Furin ${ }^{f / f l}$ mice. MF and OER wrote the manuscript with suggestions from JL, JWC, and NGS. All authors discussed the results and commented on the manuscript.

\section{Acknowledgments}

We are grateful to T.L. Clemens (Johns Hopkins School of Medicine, Baltimore, MD, USA) for providing OCN-Cre mice; S. Kousteni (Columbia University, New York, NY, USA) for providing recombinant LCN2; R. Day (Université de Sherbrooke, Sherbrooke, Canada) for providing recombinant PACE4; and A. Prat (IRCM, Montréal, Canada) for her critical reading of the manuscript.This work was supported by funding from the Canada Research Chair program (to MF and NGS); the Fondation J.A. DeSève (to MF); the Canadian Institutes of Health Research (MOP-133652, to MF, and Foundation Scheme Grant 148263, to NGS); and the Natural Sciences and Engineering Research Council of Canada (RGPIN-2016-05213, to MF). OER received scholarships from the IRCM and the Université de Montréal. JL is a fellow of the Canadian Diabetes Association.

Address correspondence to: Mathieu Ferron, Institut de Recherches Cliniques de Montréal, 110 Avenue des Pins O., Montréal, Quebec, H2W 1R7, Canada. Phone: 514.987.5754; Email: mathieu. ferron@ircm.qc.ca.

\footnotetext{
1. Karsenty G, Olson EN. Bone and muscle endocrine functions: unexpected paradigms of inter-organ communication. Cell. 2016;164(6):1248-1256.

2. Lee NK, et al. Endocrine regulation of energy metabolism by the skeleton. Cell. 2007;130(3):456-469.

3. Pi M, Wu Y, Quarles LD. GPRC6A medi-
}

ates responses to osteocalcin in $\beta$-cells in vitro and pancreas in vivo. J Bone Miner Res. 2011;26(7):1680-1683.

4. Sabek OM, Nishimoto SK, Fraga D, Tejpal N, Ricordi C, Gaber AO. Osteocalcin Effect on Human $\beta$-Cells Mass and Function. Endocrinology. 2015;156(9):3137-3146.
5. Mera P, et al. Osteocalcin Signaling in Myofibers Is Necessary and Sufficient for Optimum Adaptation to Exercise. Cell Metab. 2016;23(6):1078-1092.

6. Ferron M, McKee MD, Levine RL, Ducy P, Karsenty G. Intermittent injections of osteocalcin improve glucose metabolism and prevent type 2 diabetes in mice. Bone. 2012;50(2):568-575. 
7. De Toni L, et al. Osteocalcin and sex hormone binding globulin compete on a specific binding site of GPRC6A. Endocrinology. 2016;157(11):4473-4486.

8. Oury F, et al. Osteocalcin regulates murine and human fertility through a pancreas-bone-testis axis. J Clin Invest. 2013;123(6):2421-2433.

9. Di Nisio A, et al. The rs2274911 polymorphism in GPRC6A gene is associated with insulin resistance in normal weight and obese subjects. Clin Endocrinol (Oxf). 2017;86(2):185-191.

10. Stafford DW. The vitamin K cycle. J Thromb Haemost. 2005;3(8):1873-1878.

11. Bonneau J, et al. Association between osteocalcin gamma-carboxylation and insulin resistance in overweight and obese postmenopausal women. J Diabetes Complicat. 2017;31(6):1027-1034.

12. Ferron M, Lacombe J, Germain A, Oury F, Karsenty G. GGCX and VKORC1 inhibit osteocalcin endocrine functions. J Cell Biol. 2015;208(6):761-776.

13. Ferron $\mathrm{M}$, et al. Insulin signaling in osteoblasts integrates bone remodeling and energy metabolism. Cell. 2010;142(2):296-308.

14. Lacombe J, Karsenty G, Ferron M. In vivo analysis of the contribution of bone resorption to the control of glucose metabolism in mice. Mol Metab. 2013;2(4):498-504.

15. Jorgensen MJ, Cantor AB, Furie BC, Brown CL, Shoemaker CB, Furie B. Recognition site directing vitamin K-dependent gamma-carboxylation resides on the propeptide of factor IX. Cell. 1987;48(2):185-191.

16. Houben RJ, et al. Osteocalcin binds tightly to the gamma-glutamylcarboxylase at a site distinct from that of the other known vitamin K-dependent proteins. Biochem J. 1999;341(Pt 2):265-269.

17. Chrétien M, Mbikay M. 60 YEARS OF POMC: From the prohormone theory to pro-opiomelanocortin and to proprotein convertases (PCSK1 to PCSK9). JMolEndocrinol.2016;56(4):T49-T62.

18. Seidah NG, Prat A. The biology and therapeutic targeting of the proprotein convertases. Nat Rev Drug Discov. 2012;11(5):367-383.

19. Stijnen P, Ramos-Molina B, O'Rahilly S, Creemers JW. PCSK1 mutations and human endocrinopathies: from obesity to gastrointestinal disorders. Endocr Rev. 2016;37(4):347-371.

20. Susan-Resiga D, et al. Furin is the major processing enzyme of the cardiac-specific growth factor bone morphogenetic protein 10. J Biol Chem. 2011;286(26):22785-22794.

21. Fugère $M$, et al. Inhibitory potency and specificity of subtilase-like pro-protein convertase (SPC) prodomains. JBiol Chem. 2002;277(10):7648-7656

22. Essalmani R, et al. Furin is the primary in vivo convertase of angiopoietin-like 3 and endothelial lipase in hepatocytes. J Biol Chem. 2013;288(37):26410-26418.

23. Louagie $\mathrm{E}$, et al. Role of furin in granular acidification in the endocrine pancreas: identification of the V-ATPase subunit Ac45 as a candidate substrate. Proc Natl Acad Sci U S A. 2008;105(34):12319-12324.

24. Pesu M, et al. T-cell-expressed proprotein convertase furin is essential for maintenance of peripheral immune tolerance. Nature. 2008;455(7210):246-250.

25. Liu G, et al. Cytoskeletal protein ABP-280 directs the intracellular trafficking of furin and modulates proprotein processing in the endocytic pathway. JCell Biol. 1997;139(7):1719-1733

26. Bresnahan PA, et al. Human fur gene encodes a yeast KEX2-like endoprotease that cleaves pro-beta-NGF in vivo. J Cell Biol. 1990;111(6 Pt 2):2851-2859

27. Ratcliffe JV, Furie B, Furie BC. The importance of specific gamma-carboxyglutamic acid residues in prothrombin. Evaluation by site-specific mutagenesis. J Biol Chem. 1993;268(32):24339-24345.

28. Gundberg CM, Clough ME. The osteocalcin propeptide is not secreted in vivo or in vitro. J Bone Miner Res. 1992;7(1):73-80.

29. Roebroek AJ, et al. Failure of ventral closure and axial rotation in embryos lacking the proprotein convertase Furin. Development. 1998;125(24):4863-4876.

30. Roebroek AJ, et al. Limited redundancy of the proprotein convertase furin in mouse liver. J Biol Chem. 2004;279(51):53442-53450.

31. Zhang M, et al. Osteoblast-specific knockout of the insulin-like growth factor (IGF) receptor gene reveals an essential role of IGF signaling in bone matrix mineralization. J Biol Chem. 2002;277(46):44005-44012.

32. Kara I, et al. The paired basic amino acid-cleaving enzyme 4 (PACE4) is involved in the maturation of insulin receptor isoform $\mathrm{B}$ : an opportunity to reduce the specific insulin receptor-dependent effects of insulin-like growth factor 2 (IGF2). J Biol Chem. 2015;290(5):2812-2821.

33. Fulzele K, et al. Insulin receptor signaling in osteoblasts regulates postnatal bone acquisition and body composition. Cell. 2010;142(2):309-319.

34. Yamamoto $\mathrm{H}$, et al. Posttranslational processing of FGF23 in osteocytes during the osteoblast to osteocyte transition. Bone. 2016;84:120-130.

35. Essalmani R, et al. In vivo functions of the proprotein convertase PC5/6 during mouse development: Gdf11 is a likely substrate. Proc Natl Acad SciU S A. 2008;105(15):5750-5755.

36. Gazdag AC, Dumke CL, Kahn CR, Cartee GD. Calorie restriction increases insulin-stimulated glucose transport in skeletal muscle from IRS-1 knockout mice. Diabetes. 1999;48(10):1930-1936.

37. Mosialou I, et al. MC4R-dependent suppression of appetite by bone-derived lipocalin 2. Nature. 2017;543(7645):385-390.

38. Kim W, et al. Loss of endothelial furin leads to cardiac malformation and early postnatal death. Mol Cell Biol. 2012;32(17):3382-3391.

39. Zhu X, et al. Disruption of PC1/3 expression in mice causes dwarfism and multiple neuroendocrine peptide processing defects. Proc Natl Acad Sci U S A. 2002;99(16):10293-10298.

40. Furuta M, et al. Defective prohormone processing and altered pancreatic islet morphology in mice lacking active SPC2. Proc Natl Acad Sci U S A. 1997;94(13):6646-6651.

41. Hendy GN, Bennett HP, Gibbs BF, Lazure C, Day $\mathrm{R}$, Seidah NG. Proparathyroid hormone is preferentially cleaved to parathyroid hormone by the prohormone convertase furin. A mass spectrometric study. J Biol Chem. 1995;270(16):9517-9525.

42. Posner SF, Vaslet CA, Jurofcik M, Lee A, Seidah NG, Nillni EA. Stepwise posttranslational processing of progrowth hormone-releasing hormone (proGHRH) polypeptide by furin and PC1 Endocrine. 2004;23(2-3):199-213.

43. Himmelspach M, et al. Recombinant human factor X: high yield expression and the role of furin in proteolytic maturation in vivo and in vitro. Thromb Res. 2000;97(2):51-67.

44. Pan LC, Price PA. The propeptide of rat bone gamma-carboxyglutamic acid protein shares homology with other vitamin K-dependent protein precursors. Proc Natl Acad Sci U S A. 1985;82(18):6109-6113

45. Pan LC, Williamson MK, Price PA. Sequence of the precursor to rat bone gamma-carboxyglutamic acid protein that accumulates in warfarin-treated osteosarcoma cells. J Biol Chem. 1985;260(25):13398-13401.

46. Nishimoto SK, Price PA. The vitamin K-dependent bone protein is accumulated within cultured osteosarcoma cells in the presence of the vitamin K antagonist warfarin. J Biol Chem 1985;260(5):2832-2836.

47. Yoshikawa Y, et al. Genetic evidence points to an osteocalcin-independent influence of osteoblasts on energy metabolism. J Bone Miner Res. 2011;26(9):2012-2025.

48. Bristol JA, Freedman SJ, Furie BC, Furie B. Profactor IX: the propeptide inhibits binding to membrane surfaces and activation by factor XIa. Biochemistry. 1994;33(47):14136-14143.

49. Okun MM, Eskridge EM, Shields D. Truncations of a secretory protein define minimum lengths required for binding to signal recognition particle and translocation across the endoplasmic reticulum membrane. J Biol Chem.1990;265(13):7478-7484.

50. Demaurex N, Furuya W, D'Souza S, Bonifacino JS, Grinstein S. Mechanism of acidification of the trans-Golgi network (TGN). In situ measurements of $\mathrm{pH}$ using retrieval of TGN38 and furin from the cell surface. J Biol Chem. 1998;273(4):2044-2051.

51. Anderson ED, VanSlyke JK, Thulin CD, Jean F, Thomas G. Activation of the furin endoprotease is a multiple-step process: requirements for acidification and internal propeptide cleavage. $Е M B O$ J.1997;16(7):1508-1518

52. Isbell DT, Du S, Schroering AG, Colombo G, Shelling JG. Metal ion binding to dog osteocalcin studied by $1 \mathrm{H}$ NMR spectroscopy. Biochemistry. 1993;32(42):11352-11362.

53. Ducy P, et al. Increased bone formation in osteocalcin-deficient mice. Nature. 1996;382(6590):448-452.

54. Kingsley DM, Kozarsky KF, Hobbie L, Krieger M. Reversible defects in O-linked glycosylation and LDL receptor expression in a UDP-Gal/ UDP-GalNAc 4-epimerase deficient mutant. Cell.1986;44(5):749-759.

55. Ferron M, Wei J, Yoshizawa T, Ducy P, Karsenty G. An ELISA-based method to quantify osteocalcin carboxylation in mice. Biochem Biophys Res Commun. 2010;397(4):691-696. 